\title{
SHOCKED POSTSTARBUST GALAXY SURVEY. I. CANDIDATE POST-STARBUST GALAXIES WITH EMISSION LINE RATIOS CONSISTENT WITH SHOCKS
}

\author{
Katherine Alatalo ${ }^{1,2,8}$, Sabrina L. Cales ${ }^{3,4}$, Jeffrey A. Rich ${ }^{1,2}$, Philip N. Appleton ${ }^{2,5}$, Lisa J. Kewley ${ }^{6}$, Mark Lacy ${ }^{7}$, \\ Lauranne Lanz $^{2}$, Anne M. Medling ${ }^{6}$, and Kristina Nyland ${ }^{7}$ \\ ${ }^{1}$ Observatories of the Carnegie Institution of Washington, 813 Santa Barbara Street, Pasadena, CA 91101, USA \\ ${ }^{2}$ Infrared Processing and Analysis Center, California Institute of Technology, Pasadena, CA 91125, USA; kalatalo@carnegiescience.edu \\ ${ }^{3}$ Yale Center for Astronomy and Astrophysics, Physics Department, Yale University, New Haven, CT 06511 USA \\ ${ }^{4}$ Department of Astronomy, Faculty of Physical and Mathematical Sciences, Universidad de Concepción, Casilla 160-C, Concepción, Chile \\ 5 NASA Herschel Science Center, California Institute of Technology, Pasadena, CA 91125, USA \\ ${ }^{6}$ Research School of Astronomy and Astrophysics, Australian National University, Cotter Road, Weston ACT 2611, Australia \\ 7 National Radio Astronomy Observatory, 520 Edgemont Road, Charlottesville, VA 22903, USA \\ Received 2015 June 17; accepted 2016 April 13; published 2016 June 17
}

\begin{abstract}
There are many mechanisms by which galaxies can transform from blue, star-forming spirals, to red, quiescent early-type galaxies, but our current census of them does not form a complete picture. Recent observations of nearby case studies have identified a population of galaxies that quench "quietly." Traditional poststarburst searches seem to catch galaxies only after they have quenched and transformed, and thus miss any objects with additional ionization mechanisms exciting the remaining gas. The Shocked POststarburst Galaxy Survey (SPOGS) aims to identify transforming galaxies, in which the nebular lines are excited via shocks instead of through star formation processes. Utilizing the Oh-Sarzi-Schawinski-Yi (OSSY) measurements on the Sloan Digital Sky Survey Data Release 7 catalog, we applied Balmer absorption and shock boundary criteria to identify 1067 SPOG candidates $\left(\mathrm{SPOGs}^{*}\right.$ ) within $z=0.2$. SPOGs ${ }^{*}$ represent $0.2 \%$ of the OSSY sample galaxies that exceed the continuum signal-to-noise cut (and $0.7 \%$ of the emission line galaxy sample). SPOGs* colors suggest that they are in an earlier phase of transition than OSSY galaxies that meet an "E+A" selection. SPOGs* have a $13 \% 1.4 \mathrm{GHz}$ detection rate from the Faint Images of the Radio Sky at Twenty Centimeters Survey, higher than most other subsamples, and comparable only to low-ionization nuclear emission line region hosts, suggestive of the presence of active galactic nuclei (AGNs). SPOGs* also have stronger Na I D absorption than predicted from the stellar population, suggestive of cool gas being driven out in galactic winds. It appears that SPOGs* represent an earlier phase in galaxy transformation than traditionally selected poststarburst galaxies, and that a large proportion of SPOGs* also have properties consistent with disruption of their interstellar media, a key component to galaxy transformation. It is likely that many of the known pathways to transformation undergo a SPOG phase. Studying this sample of SPOGs* further, including their morphologies, AGN properties, and environments, has the potential for us to build a more complete picture of the initial conditions that can lead to a galaxy evolving.
\end{abstract}

Key words: catalogs - galaxies: active - galaxies: evolution - galaxies: statistics

Supporting material: extended figure, machine-readable table

\section{INTRODUCTION}

Galaxies exhibit a prominent bimodality in color, morphology, star formation (SF) rates, and stellar mass space (Hubble 1926; Baade 1958; Holmberg 1958; Tinsley 1978; Kauffmann et al. 2003; Blanton \& Moustakas 2009). The color-magnitude diagram is a manifestation of this bifurcation, depicting a blue cloud and a red sequence (Tinsley 1978; Strateva et al. 2001; Baldry et al. 2004). The blue cloud consists of a population of galaxies that are actively starforming, gas-rich, and disk-dominated, while the red sequence is typically populated by quiescent, gas-poor galaxies with spheroidal morphologies. The total mass of blue cloud objects has remained roughly constant over the last $\sim 6 \mathrm{Gyr}$ (from $z=1$ $\rightarrow 0$ ), forming stars at a rate that maintains constant SF ratestellar mass relation, sitting on the so-called "SF main sequence" (Noeske et al. 2007; Wuyts et al. 2011). The red sequence on the other hand has doubled in mass (Bell et al. 2007, 2012), suggesting that a non-negligible fraction

\footnotetext{
8 Hubble fellow.
}

of blue cloud galaxies have quenched their SF and evolved to the red sequence. The transformation from blue to red at $z=0$ also appears to be one-way (Appleton et al. 2014; Young et al. 2014), barring an extreme external event, such as a gas-rich merger (Kannappan et al. 2009; McIntosh et al. 2014). Given the multitude of pathways to red sequence, it is vitally important to understand all the initial conditions that catalyze this transformation.

Galaxies located in the sparsely populated region between the blue cloud and red sequence, the so-called "green valley," were hypothesized to be taking part in a rapid transition (Faber et al. 2007). A recent in-depth study by Schawinski et al. (2014) of the morphologies of galaxies within the green valley found that while early-type galaxies (ETG) do indeed transition rapidly ( $\approx 100 \mathrm{Myr}$ ) through the green valley, late-type galaxies (LTGs) do not. They conclude that green colors alone are insufficient to identify transitioning galaxies since LTGs inherit their green colors from a substantial buildup of an older stellar population over the course of many Gyrs while maintaining a near-constant SF rate. 


\subsection{Star Formation Quenching Mechanisms}

Many evolutionary paths to transform a blue spiral into a red elliptical have been hypothesized. Lilly et al. (2013) describes a simple picture for the evolution of a typical galaxy, beginning with a blue galaxy on the star-forming main sequence, increasing in mass by accreting gas from the cosmic web and through mergers. Once critical mass is reached, the gas supply is cut off, quenching the SF, and the galaxy evolves onto the red sequence. This implies that the halo (bulge) grew sufficiently large to stabilize against gravitational collapse, inhibiting SF (Kauffmann et al. 2003; Cattaneo et al. 2006; Martig et al. 2009, 2013; Davis et al. 2014).

Peng et al. (2010) suggest that in addition to morphology, environment also contributes to regulating SF. As a galaxy falls into a cluster environment, it experiences ram pressure stripping, in which the intracluster medium strips the less dense galactic interstellar medium (ISM; Gunn \& Gott 1972; Chung et al. 2009). The hot intracluster medium also plays a role in inhibiting the accretion of new neutral material (strangulation; Bekki et al. 2002; Davis et al. 2011), inhibiting new star-forming fuel from reaching the galaxy and forming stars. In the process known as harassment (high-speed fly-bys in clusters), perturbations in a galaxy's gravitational potential can affect the efficiency of SF (Mihos 1995; Moore et al. 1996; Bekki 1998).

Recently, a comprehensive study of five clusters between $0.31<z<0.54$ by Dressler et al. (2013) called into question whether the majority of galaxy transformation takes place after the galaxies have been accreted by and virialized with the cluster, instead suggesting that group pre-processing (during the fall-in group phase) plays a larger role in the late-type to early-type transitions than the cluster itself. In fact, tidal stripping and harassment are also at play in compact groups (Hickson 1997; Verdes-Montenegro et al. 2001; Rasmussen et al. 2008; Sivanandam et al. 2010), and observational evidence suggests that compact group galaxies evolve rapidly (Johnson et al. 2007; Walker et al. 2010), thus the possibility that cluster galaxies could be transformed in the fall-in group phase rather than virialized within the cluster is plausible.

In the field, mergers and interactions play a prominent role in galaxy evolution (Toomre \& Toomre 1972; Springel et al. 2005; Hopkins et al. 2006), transforming disks to spheroids, driving massive amounts of gas to nuclear regions, and triggering SF and black hole growth. This activity rapidly depletes available gas stores via the starburst and can trigger feedback from the active galactic nucleus (AGN; Di Matteo et al. 2005; Feruglio et al. 2010; Cicone et al. 2014, 2015), quenching SF. Observational evidence supports the merger hypothesis: the most luminous AGNs tend to be produced by mergers while less luminous AGNs are mainly powered by secular processes (Treister et al. 2012). AGNs and SF can also provide negative feedback that regulates SF. Radiative-mode AGNs can cause ionization, heating, and radiation pressure that may regulate SF (Ciotti \& Ostriker 2007; Cano-Díaz et al. 2012). Mechanical-mode AGNs that drive nuclear winds and jets can remove fuel for SF from the galaxy (Bîrzan et al. 2008; Ciotti et al. 2010; Alatalo et al. 2011; Nyland et al. 2013), though recent simulations and observations seem to indicate AGN feedback mechanisms at $z=0$ do not provide the global SF quenching that was expected from previous simulations (Springel et al. 2005), but rather act quite locally $(\lesssim 1 \mathrm{kpc})$ to the supermassive black hole (Costa et al. 2014;
Gabor \& Bournaud 2014; García-Burillo et al. 2014; Alatalo 2015). However, there is evidence that quasars at high $-z$ produce the extreme energies required to change the global properties of their hosts (Zakamska et al. 2016).

\subsection{Quiet Quenching}

The nearby, low ionization nuclear emission line region (LINER; Kewley et al. 2006) galaxy, NGC 1266, although unremarkable in its ground-based optical appearance, was found to be hosting a dramatic AGN-driven multiphase outflow, and a $10^{9} M_{\odot}$ reservoir of molecular gas contained in the nucleus (Alatalo et al. 2011). Upon deeper inspection, NGC 1266 was found to contain shocked ionized gas ratios associated with the outflow (Davis et al. 2012), a Comptonthick AGN (Nyland et al. 2013; Alatalo et al. 2015b), and an intermediate stellar population (1/2 Gyr; Alatalo et al. 2014c). The SF within the molecular core of the galaxy was also found to be severely suppressed, leading to an inefficiency of $\gtrsim 50$ (Alatalo et al. 2015b), likely due to the turbulence being injected into the system by the powerful shocks associated with the outflow, thereby extending the lifetime of the nuclear molecular gas by two orders of magnitude. Alatalo et al. (2014c) concluded that the observational properties of NGC 1266 were likely caused by a minor merger that drove gas to the nuclear region of the galaxy and triggered a burst of SF but that these events occurred $1 / 2 \mathrm{Gyr}$ ago, and have left few signs of the initial triggering event in the galaxy. Ultimately, the molecular disk fueled the black hole, which formed a low-power radio jet that quenched the initial starburst and continues to inhibit SF through turbulence, rather than directly expelling the gas from the halo (Alatalo et al. 2015b).

Indeed the recent discoveries into the nature of NGC 1266, a galaxy undergoing substantial transformation and SF quenching, suggests that there is a population of objects that have not previously been identified as transforming, due to their lack of obvious clues such as tidal tails, starburst signatures, or prominent AGN activity. We call this particular pathway "quiet quenching." NGC 1266 is an ideal case study for this new, quietly quenching population, which transform from spirals into ellipticals in spite of there being no outward, obvious signs of the process. Thus, galaxies with poststarburst spectral signatures and shock-like ionized gas line ratios may comprise an important, overlooked segment of the transitioning galaxy population, containing exactly those objects which are being actively impacted by turbulence.

\subsection{Shocked Poststarburst Galaxies (SPOGs)}

Many of the outlined mechanisms that lead to the transformation of a galaxy are violent, and thus are accompanied by shocks that inject turbulence into the starforming gas, heating it and serving as a catalyst for its expulsion, ultimately quenching SF in the form of a massive galactic wind (Armus et al. 1990; Heckman et al. 1990; Rich et al. 2010; Sturm et al. 2011). Shocks are prevalent in merging systems (Rich et al. 2011, 2014; Peterson et al. 2012; Soto et al. 2012; Inami et al. 2013), in galaxies located in the outskirts of clusters (Braglia et al. 2009; Sivanandam et al. 2010; Cen et al. 2014), and galaxy groups (Appleton et al. 2006, 2013; Cluver et al. 2010, 2013; Vogt et al. 2013, 2015). Shocks are also a ubiquitous feature of galactic winds that are being driven by quasars (Nesvadba et al. 2008; Harrison et al. 2014; Villar 
Martín et al. 2014). For transitioning galaxies in both the modern and early universe, shocks serve as a beacon pinpointing those galaxies that are in the most dramatic phases of their evolution.

One classical way that galaxies have been identified as transitioning objects is by searching for poststarburst galaxies (Dressler \& Gunn 1983; Zabludoff et al. 1996), which stood out based on their disparate emission line and stellar population characteristics. Poststarburst galaxies are identified based on the presence of an intermediate age (A-star) stellar population and the absence of emission lines consistent with SF (using nebular lines such as $\mathrm{H} \alpha$ or [O II] $\lambda 3727 \AA$; Quintero et al. 2004; Goto 2005, 2007). This disparate set of characteristics demonstrates that these objects are a subset that had a recent burst of SF that has ceased rapidly. This type of search was used as the benchmark for finding transitioning objects, and yet a classical poststarburst search would miss the massive molecular outflow host NGC 1266 due to its shock-powered $\mathrm{H} \alpha$ emission (Davis et al. 2012; Alatalo et al. 2014c).

New studies expanding the selection criteria for poststarburst galaxies have called into question the reasoning behind the limit placed on nebular emission (Falkenberg et al. 2009; Yesuf et al. 2014). In particular, placing a limit on nebular emission lines introduces a bias against AGNs (Wild et al. 2009; Cales et al. 2011, 2013; Kocevski et al. 2011; Alatalo et al. 2014b) because AGNs can power significant emission of [O II] and $\mathrm{H} \alpha$. Placing a limit on nebular emission biases the poststarburst selection against any quenching objects that contain energetic phenomena (such as AGNs or shocks) capable of exciting those lines. Furthermore, an [O II] limit also produces a metallicity bias, since $[\mathrm{O} \mathrm{II}]$ is weak at both very high and very low metallicities (Kewley et al. 2004).

A secondary limitation of the poststarburst search is that by requiring the absence of $[\mathrm{O} \mathrm{II}]$ and $\mathrm{H} \alpha$, it is only able to identify galaxies after all SF has ceased, rather than at the point SF is abruptly diminishing, increasing the time between identification and the suspected preceding starburst phase (Hogg et al. 2006). Snyder et al. (2011) investigated the quenching process and the poststarburst phase in a suite of simulations, which showed substantial scatter in the total time of the $\mathrm{K}+\mathrm{A}$ phase, but also significant dependencies on many of the initial conditions of the merger. By the time the galaxy has a detectable $\mathrm{K}+\mathrm{A}$ phase, many of the initial conditions associated with the triggering event have faded. Therefore, identifying galaxies at an earlier phase of transition is also important to a complete understanding of how galaxies quench, and transform from blue to red.

If quenching galaxy searches reject NGC 1266-like galaxies, they may miss a substantial population of transitioning galaxies. Given that, at $z=0$, the evolutionary path from blue spiral to red early-type is one-way (Appleton et al. 2014; Young et al. 2014), it is essential to understand all initial conditions that could lead to this transition, including the population that has quietly quenched. Robustly measuring the fraction of quiet quenchers occurring in the overall galaxy population will provide an estimate of their duty cycle, and studying them in detail will provide insights into the connections between the source of the narrow line emission and the quenching of SF. To this end, we have created the Shocked POststarbust Galaxy Survey $\left(\right.$ SPOGS $\left.^{9}\right)$, building a

\footnotetext{
http://www.spogs.org
}

catalog of poststarburst galaxies hosting narrow line ratios consistent with shocks. This catalog focuses on Sloan Digital Sky Survey (SDSS) DR7 (Abazajian et al 2009) ${ }^{10}$ galaxies with $z<0.2$ and utilizes the Oh-Sarzi-Schawinski-Yi absorption and emission line catalog (OSSY; Oh et al. 2011). ${ }^{11}$

This paper is part of a series dedicated to studying the connection between the total emission-line galaxy (ELG) population and the intersection of transitioning galaxies and those with shock line ratios. In Section 2, we lay out the selection criteria for the SPOG sample and present the parent sample. In Section 3, we discuss the role SPOGs play in the context of galaxy evolution and lay the groundwork for deeper investigations of SPOGs. In Section 4, we summarize our results. The cosmological parameters $H_{0}=70 \mathrm{~km} \mathrm{~s}^{-1}$, $\Omega_{m}=0.3$, and $\Omega_{\Lambda}=0.7$ (Spergel et al. 2007) are used throughout.

\section{SPOGS SAMPLE DEFINITION AND CHARACTERISTICS}

\subsection{The ELG Catalog}

To build a catalog of SPOG candidates (deemed SPOGs*), we drew from the OSSY sample, a comprehensive database of the emission, absorption, and continuum measurements of 664,187 SDSS Data Release 7 (DR7; Abazajian et al 2009) galaxies within $z=0.2 .^{12}$ OSSY uses penalized pixel-fitting algorithms (PPXF; Cappellari \& Emsellem 2004) to fit stellar population templates and the absorption features and kinematics in combination with an algorithm that fits the absorption and emission lines (GANDALF; Sarzi et al. 2006).

Here we describe some of the fit and quality assurance parameters from the OSSY measurements (Oh et al. 2011). The signal, statistical noise, and residual noise are measured using the averages of the continuum bands $4500-4700 \AA$, $5400-5600 \AA, 6000-6200 \AA$, and $6800-7000 \AA$. The ratio of the fit residuals (i.e., residual noise, $r N$ ) to statistical fluctuations $(s N)$ corresponds to the reduced $\chi^{2}$ statistical measurement and is used to assess the quality of the model. When binned by the signal-to-noise ratio $(\mathrm{S} / \mathrm{N})$ this goodnessof-fit parameter $(r N / s N)$ centers around unity with a distribution of values above and below this value of standard deviation, $\sigma$. Objects far from unity, as measured by the number of $\sigma$ from the median $r N / s N\left(N_{\sigma}\right)$, have particularly poor fits.

The parent ELG catalog is a subset of the OSSY catalog that contains all emission line galaxies that satisfy our continuum and emission-line $\mathrm{S} / \mathrm{N}$ criteria. To build the parent sample, we limited the ELG catalog to data from the OSSY database meeting the following two quality assurance criteria in continuum:

1. $\mathrm{S} / \mathrm{N}>10$, to ensure robust absorption feature detection

2. $N_{\sigma}<3$, which limits the ratio of residual to statistical noise in units of standard deviations from the median.

591,627 (89\%) galaxies meet the $\mathrm{S} / \mathrm{N}$ continuum criteria. We additionally applied the following quality assurance criteria for all the following narrow lines: $\mathrm{H} \beta,[\mathrm{O} \mathrm{III}], \mathrm{H} \alpha,\left[\mathrm{N}_{\mathrm{II}}\right],\left[\mathrm{S}_{\mathrm{II}}\right]$, and $[\mathrm{O} \mathrm{I}]$ :

\footnotetext{
${ }^{10}$ http://classic.sdss.org/dr7/

11 http://gem.yonsei.ac.kr/ ksoh/wordpress/

12 Approximately $7 \%$ of SDSS objects have multiple spectra in the SDSS database, so the spectra do not necessarily represent unique objects.
} 
Table 1

Line Diagnostic Type

\begin{tabular}{lrr}
\hline \hline Type & Number & Percentage $^{\mathrm{a}}$ \\
\hline Ambiguous & 17,097 & $10.7 \pm 0.07$ \\
SF & 111,972 & $70.2 \pm 0.11$ \\
Composite & 14,226 & $8.9 \pm 0.07$ \\
Seyfert & 4765 & $3.0 \pm 0.04$ \\
LINER & 11,327 & $7.2 \pm 0.06$ \\
\hline Total & 159,387 & 100.0 \\
\hline
\end{tabular}

Note.

${ }^{a}$ Includes binomial errors contribute negligibly.

1. $\mathrm{A} / \mathrm{N}>1$, peak line flux (amplitude) to noise

2. Line $N_{\sigma}<5$, which limits the ratio of residual to statistical noise in units of standard deviations from the median

These criteria ensure that our parent sample includes only robust detections of both absorption and emission lines, and allows us to classify galaxies over the entire suite of line diagnostics $\left([\mathrm{O} \mathrm{III}] / \mathrm{H} \beta\right.$ versus $[\mathrm{N} \mathrm{II}] / \mathrm{H} \alpha$, $\left[\mathrm{S} \mathrm{II}_{\mathrm{II}}\right] / \mathrm{H} \alpha$, and $\left[\mathrm{O}_{\mathrm{I}}\right] /$ $\mathrm{H} \alpha$ ), reducing ambiguity. A total of 159,387 galaxies $(24 \pm 0.05 \%)$ of the OSSY sample pass all of our quality control criteria and are considered part of the parent sample. The ELG sample was then sub-classified based on their line diagnostic ratios (Baldwin et al. 1981; Veilleux \& Osterbrock 1987) as either Seyferts, LINERs, composites, starforming or ambiguous (in which an object did not fit into a single classification across all three line diagnostics) based on the models and empirical classification lines of Kewley et al. (2006). Table 1 and Figure 1 show the fractional representation of each line diagnostic state found in the ELG sample. It is clear that the majority of objects that passed quality control (and thus had strong line emission) are star-forming galaxies.

\subsection{Stellar Population Criterion}

Classically a poststarburst galaxy is defined by Dressler \& Gunn (1983) as one that exhibits strong Balmer absorption lines, indicating intense SF in the past $\sim \mathrm{Gyr}$, and a lack of ongoing SF, as indicated by having little or no nebular emission (usually chosen to be either the $\mathrm{H} \alpha$ or $[\mathrm{O}$ II] $\lambda 3727 \AA$ line). Their spectra are best modeled as a superposition of an elliptical galaxy-like spectrum and an A-star spectrum, hence they are often called " $\mathrm{E}+\mathrm{A}$ " or " $\mathrm{K}+\mathrm{A}$ " galaxies (Quintero et al. 2004). Balmer absorption is strongest in A-type stars (Vazdekis et al. 2010), and thus is often used as a tracer of a stellar population that was formed in a recent burst of SF. Falkenberg et al. (2009) calculated a grid of galaxy models with different combinations and a sudden increase or decrease of the SF rate on different timescales and find that a poststarburst phase can be induced via starbursts, or an abrupt termination of SF. We have chosen the Balmer line, $\mathrm{H} \delta$, for our cut, since it is neither contaminated by absorption lines (such as $\mathrm{H} \epsilon$, which falls within the $\mathrm{Ca}$ II $\mathrm{H}$ absorption trough), nor substantially filled in by nebular emission lines (as is common in $\mathrm{H} \alpha$ and non-negligible in $\mathrm{H} \beta$ and $\mathrm{H} \gamma$ ). The OSSY catalog uses emission line infilling-subtracted absorption line fits to mitigate the effects of infilling, though there likely still persist biases against galaxies with strong emission lines, such as AGNs, and toward more highly reddened sources. We set a

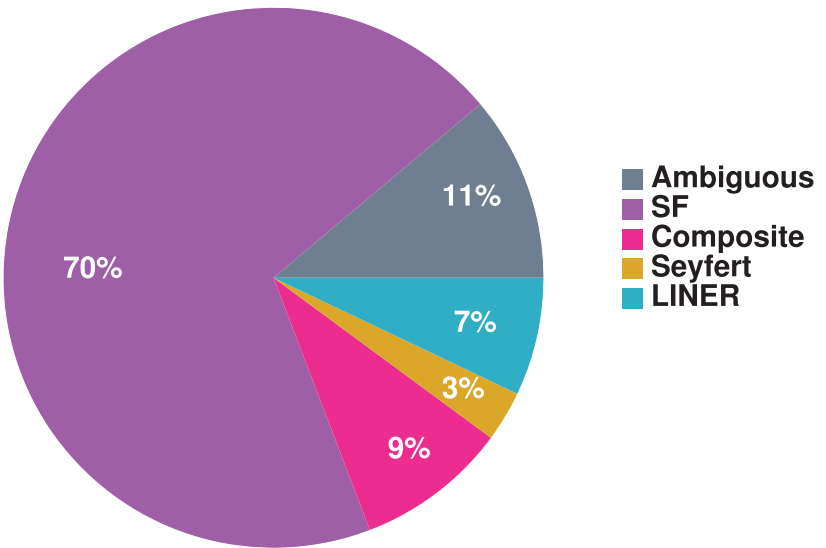

Figure 1. The fractional representation of objects in the emission line galaxy (ELG) sample. The majority $(70 \%)$ of galaxies in the ELG contain line diagnostic ratios consistent with star formation.

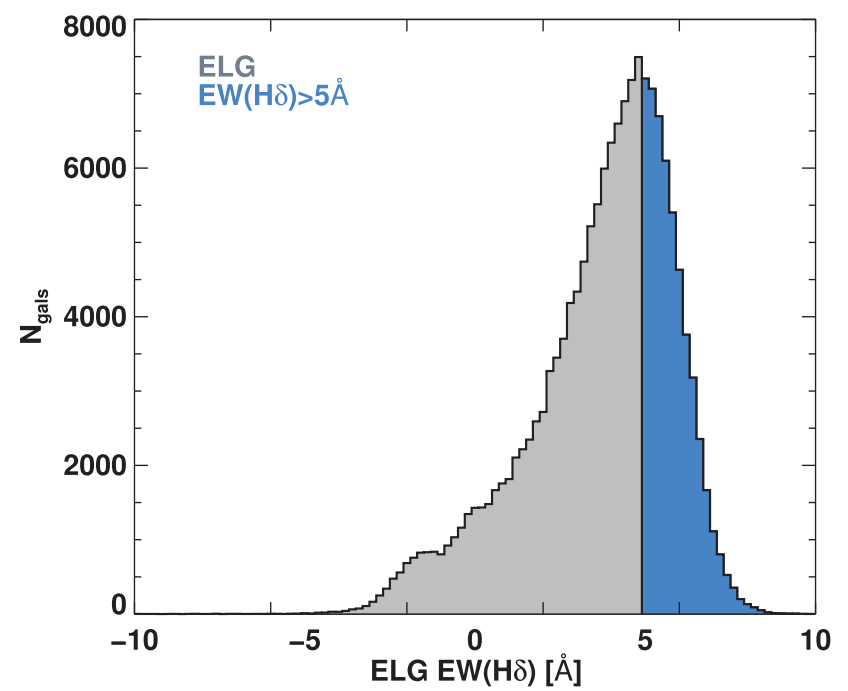

Figure 2. The distribution of $\mathrm{EW}(\mathrm{H} \delta)$ values in the ELG sample. $29 \%$ of galaxies in the ELG sample have $\operatorname{EW}(\mathrm{H} \delta)>5 \AA$, shaded in blue.

threshold of $\mathrm{EW}(\mathrm{H} \delta)>5 \AA$, consistent with the Balmer poststarburst criteria of Goto (2007) and Falkenberg et al. (2009).

Figure 2 shows the 46,936 (29 $\pm 0.11 \%)$ objects in the ELG that have $\mathrm{EW}(\mathrm{H} \delta)>5 \AA$. The majority of sources in the ELG sample have line diagnostics consistent with SF (Figure 1). Alatalo et al. (2014b) also showed that most sources with $\mathrm{EW}(\mathrm{H} \delta)>5 \AA$ had very blue colors. In fact, 44,327 $(94 \pm 0.10 \%)$ of the galaxies that fit the $\mathrm{EW}(\mathrm{H} \delta)>5 \AA$ criteria also lie in the star-forming region of the line diagnostic diagrams. This is indicative of a population that is continuously forming stars, having built a substantial intermediate stellar population along the way.

\subsection{Colors and Stellar Masses}

We use the dereddened magnitudes from SDSS DR9 (Ahn et. al 2012) (which are corrected for Galactic extinction) for our bandpasses. The $u-r$ colors are then $k$-corrected using the IDL routine calc_kcor ${ }^{13}$ (Chilingarian \& Zolotukhin 2012), and

\footnotetext{
13 http://kcor.sai.msu.ru/
} 
corrected for intrinsic extinction using the stellar $E(B-V)$ values from the OSSY catalog (Oh et al. 2011). The stellar masses were determined from the $k$-corrected, extinction corrected $i$-band dereddened fluxes and $u-r$ colors, using the $M_{i}-u-r$ to stellar mass conversion from Bell et al. (2003).

\subsection{Defining the Shock Boundaries}

To search for shocked gas in galaxies, we rely on grids of shock models generated with MAPPINGS III (Dopita \& Sutherland 1995, 1996; Dopita et al. 2005; Allen et al. 2008; Rich et al. 2011). Figure 3 show the distribution of fast (black points; Allen et al. 2008) and slow (colored lines; Rich et al. 2011) shock model grids in the line diagnostic diagrams with log $([\mathrm{O} \mathrm{III}] / \mathrm{H} \beta)$ versus $\log ([\mathrm{N} \mathrm{II}] / \mathrm{H} \alpha), \log \left(\left[\left[\mathrm{S}_{\mathrm{II}}\right] / \mathrm{H} \alpha\right)\right.$, and $\log$ $([\mathrm{O} \mathrm{I}] / \mathrm{H} \alpha)$ optical emission line ratios. The fast shock grids cover a much broader range of velocities (from $100-1000 \mathrm{~km} \mathrm{~s}^{-1}$ ), with the fast shock models (Dopita et al. 2005; Allen et al. 2008) covering $1 Z_{\odot}$ and $2 Z_{\odot}$ metallicities, and slow shock models covering 1,2 , and $3 Z$. metallicities. $[\mathrm{O} \mathrm{I}] / \mathrm{H} \alpha$ is a particularly good tracer of shock excitation (Farage et al. 2010; Rich et al. 2010). The possible line ratios cover a wide excitation space, and we have used these distributions to define a polygon that encompasses galaxy emission-line ratios consistent with shock excitation (Figure 3). The area covered by these polygons also overlaps regions traditionally associated with other emission-line processes including LINER, Seyfert, and $\mathrm{H}$ II region emission (Kauffmann et al. 2003; Kewley et al. 2006). This is unavoidable, but is not unexpected given observations of shocks in nearby galaxies (e.g., Rich et al. 2011; Ho et al. 2014). The shock criteria and boundaries are defined based on the following equations: Shock criteria:

$$
\begin{aligned}
-0.75<\log ([\mathrm{N} \mathrm{II}] / \mathrm{H} \alpha) & <0.42 \\
-0.81<\log ([\mathrm{S} \mathrm{II}] / \mathrm{H} \alpha) & <0.44 \\
-2.06<\log ([\mathrm{O} \mathrm{I}] / \mathrm{H} \alpha) & <0.34 \\
-0.81<\log ([\mathrm{O} \mathrm{III}] / \mathrm{H} \beta) & <1.03
\end{aligned}
$$

and functions:

$$
\begin{aligned}
& \log ([\mathrm{O} \mathrm{III}] / \mathrm{H} \beta)> 0.4 /[\log ([\mathrm{N} \mathrm{II}] / \mathrm{H} \alpha)+0.15] \\
&+\log ([\mathrm{N} \mathrm{II}] / \mathrm{H} \alpha)+1.5 \\
& \log ([\mathrm{O} \mathrm{III}] / \mathrm{H} \beta)>0.65 \log ([\mathrm{N} \mathrm{II}] / \mathrm{H} \alpha)-0.62 \\
& \log ([\mathrm{O} \mathrm{III}] / \mathrm{H} \beta)<1.12 \log ([\mathrm{N} \mathrm{II}] / \mathrm{H} \alpha)+1.14 \\
& \log ([\mathrm{O} \mathrm{III}] / \mathrm{H} \beta)>1.05 /[\log ([\mathrm{S} \mathrm{II}] / \mathrm{H} \alpha)-1.00] \\
&+0.5 \log ([\mathrm{S} \mathrm{II}] / \mathrm{H} \alpha)+0.74 \\
& \log ([\mathrm{O} \mathrm{III}] / \mathrm{H} \beta)>1.15 /[\log ([\mathrm{O} \mathrm{I}] / \mathrm{H} \alpha)-0.95] \\
&-0.15 \log ([\mathrm{O} \mathrm{I}] / \mathrm{H} \alpha)+0.30
\end{aligned}
$$

30,225 (19\%) of the ELGs catalog fall within these shock model boundaries across all three line diagnostic diagrams. We note that there is significant overlap of the shock boundaries with SF, composites, Seyferts and LINERs, and therefore we do not expect the majority of galaxies that fall within the shock boundaries to be dominated by shocks. Furthermore, we do not expect galaxies to harbor a single source of ionization, rather that they could harbor shocks as well as additional sources of ionization. Seyferts with $\log ([\mathrm{O} \mathrm{III}] / \mathrm{H} \beta)>1.03$ can be distinguished from shocks; however, even a large portion of quasars with poststarburst signatures fall below the traditional Seyfert
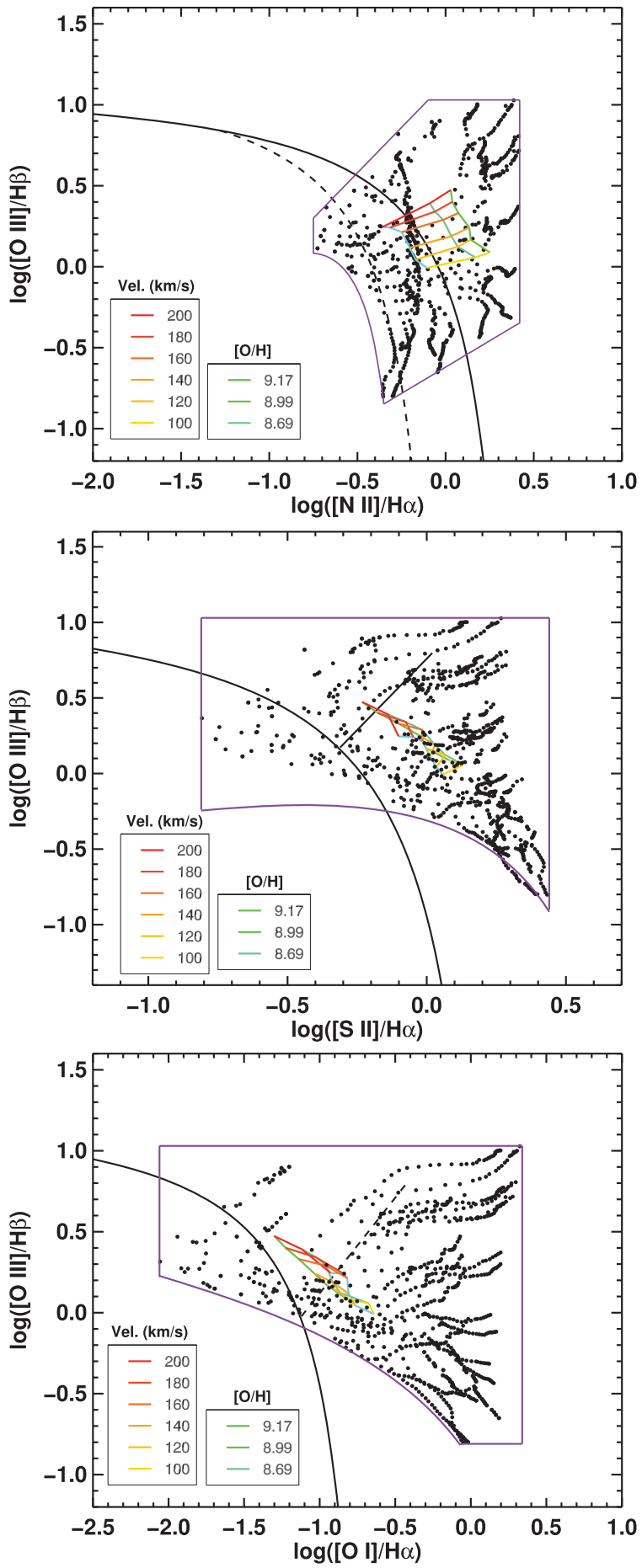

Figure 3. Line diagnostic diagrams (Baldwin et al. 1981; Veilleux \& Osterbrock 1987) for $[\mathrm{O} \mathrm{III]} / \mathrm{H} \beta$ vs. [N II] $/ \mathrm{H} \alpha$ (top), [S II] $/ \mathrm{H} \alpha$ (middle), and $\left[\mathrm{O}_{\mathrm{I}}\right] / \mathrm{H} \alpha$ (bottom) with both fast (black points; Allen et al. 2008) and slow (colored grids; Rich et al. 2011) shock models with various metallicities, and shock velocities ranging from 100 to $1000 \mathrm{~km} \mathrm{~s}^{-1}$, overlaid with the star formation boundary (black line; Kewley et al. 2006, dashed line in [N II] $/ \mathrm{H} \alpha$; Kauffmann et al. 2003). The fast shock grids cover a much broader range of velocities, resulting in a correspondingly larger range of permissible emission line ratios. The purple line defines the boundaries put in place to encompass the entire phase space of the shock models (defined in Section 2.4). These models show that shocks can be located in all regions of the diagnostic diagrams, mimicking line ratios associated with SF, composites, Seyferts, and LINERs. 
cutoff of $\log ([\mathrm{O} \mathrm{III}] / \mathrm{H} \beta)>1.03$ and therefore also within the shock boundaries (Cales et al. 2013).

LINER emission accounts for the majority of phase space defined within the shock boundaries, and the largest portion $(36 \%)$ of shock candidates from the ELG sample. Seyferts account for the next largest subset (14\%), followed by composites (6\%), and SF (4\%). The remaining $40 \%$ of shock candidates have ambiguous line diagnostic classifications (fairly consistent with the $43 \%$ of shock models that produce ambiguous classifications; Dopita et al. 2005; Allen et al. 2008; Rich et al. 2011). The requirement that shock candidates must be found within the shock boundaries within all three line diagnostic diagrams mitigates the risk of significant contamination from the SF region, which comprises the majority of the ELG sample.

\subsection{Prevalence of Star Forming Contaminants}

A total of 4508 (3\%) objects have line diagnostics consistent with shocks and Balmer absorption, indicative of a strong A-star population $(\mathrm{EW}(\mathrm{H} \delta)>5 \AA)$. We note that a large number $(3441,76 \%)$ of these systems have narrow line emission diagnostics matching models of SF. For this reason, to define a sample of pure poststarburst, shocked candidates, we remove all galaxies with $\mathrm{EW}(\mathrm{H} \delta)>5 \AA$ that consistently fall within the Composite or SF region of the line diagnostic diagrams (Kewley et al. 2006) for all three diagnostic diagrams. While it is possible that we are missing very interesting galaxies that fall in the region where SF and shocks overlap, the data in-hand do not allow us to distinguish between the true shocked galaxies and star-forming galaxies.

The properties of the shock models that fall within the SF region of the line diagnostic diagrams tend to be those with low metallicities and higher densities, and thus are missed from our shock definition. There is also the possibility of contamination of "red bulge" galaxies. Below $z=0.016$, the 3" SDSS fiber covers less than $1 \mathrm{kpc}$ diameter. If the $3^{\prime \prime}$ SDSS fiber only covers the nucleus of a bulge-dominated spiral, it could be classified as a shock candidate, despite the fact that the integrated emission from the galaxy would be clearly classified as star-forming. In 55 SPOGs ${ }^{*}$, less than $10 \%$ of the optical $i$ band light went down the fiber (calculated by taking the ratio of the fiber and Petrosian magnitudes). Many of these were at very low redshift $(z<0.01)$, but others appeared to be due to offset fiber positions. We inspected these objects; while some were contaminants, many others showed signs of being real SPOGs. For completeness, we did not remove these 55 SPOGs* from the catalog.

To determine the contamination fraction of "red bulge" galaxies (galaxies that contained extended star-forming disks and quiescent nuclei) that were not covered by the $3^{\prime \prime}$ SDSS fiber, we manually inspected all identified SPOGs* with redshifts below $z<0.03$ (a total of 109 objects), identifying galaxies with clear signatures of SF outside of the nucleus. Most SPOG candidates $(81 \%)$ below $z<0.01$ exhibited signs of extended SF in their outer disks. The contamination fraction reduces rapidly with increasing redshift increments $34 \%$ at $0.025 \leqslant z<0.03$, down to $4 \%$ at $0.04 \leqslant z<0.06$ ). The SPOG selection has strongly redshift-dependent contamination effects, although we did not remove these objects from the survey to remain complete in our criterion selection. We recommend those selecting the lowest redshift SPOGs* to carefully inspect those objects. A more detailed discussion of biases within the
ELG and SPOG* sample can be found in the Appendix. A more detailed description of the morphologies of SPOGs is beyond the scope of this paper.

\subsection{The Shocked Poststarburst Galaxy (SPOG) Sample}

We define the Shocked Poststarburst Galaxy (SPOG) sample as objects from the ELG catalog that simultaneously meet the $\mathrm{EW}(\mathrm{H} \delta)>5 \AA$ and shocked criteria, while also not falling consistently inside the SF and composite regions of all three line diagnostic diagrams. 1067 objects $(0.7 \%$ of the 159,387 ELGs) meet this criterion. Given that a location within the shock boundaries does not confirm the presence of shocks, we identify these objects as candidates, or SPOGs*. We visually inspected the thumbnails for each $\mathrm{SPOG}^{*}$ to ensure that no galaxies included duplicate spectra, and found that in all cases, each SPOG-identified spectrum represented a single object.

Figure 4 shows the $\left[\mathrm{O}\right.$ III] $/ \mathrm{H} \beta$ versus $\left[\mathrm{N}\right.$ II] $/ \mathrm{H} \alpha,\left[\mathrm{S}_{\mathrm{II}}\right] / \mathrm{H} \alpha$, and $\left[\mathrm{O}_{\mathrm{I}}\right] / \mathrm{H} \alpha$ line diagnostic diagrams (Baldwin et al. 1981; Veilleux \& Osterbrock 1987) for the entire ELG sample (grayscale), with the SPOGs* plotted as green points. The shock boundaries are shown as purple lines, and the different line ratio population divisions (Kauffmann et al. 2003; Kewley et al. 2006) are shown as black lines. SPOGs* span the range defined by the shock boundaries in Section 2.4 and contain nearly equal contributions from objects classed as LINERs $(186 ; 17 \pm 1.1 \%)$ and Seyferts $(194 ; 18 \pm 1.2 \%)$, with the remainder having ambiguous classifications.

To test how significantly aperture bias affects the catalog, we plot the distribution of SDSS absolute $r$-band magnitude $\left(M_{r}\right.$, corrected for Galactic extinction) versus $z$ in Figure 5 for the objects that have been spectrally classified as ambiguous, composite, SF, Seyfert, and LINER; objects meeting the $\mathrm{EW}(\mathrm{H} \delta)>5 \AA$ criteria; those within the shock boundaries; and SPOGs*. In $M_{r-z}$ space, SPOGs* follow similar trends to starforming galaxies and $\mathrm{EW}(\mathrm{H} \delta)>5 \AA$ objects, rather than LINER or shock-boundary objects, populating the low $M_{r}$ region at low redshift (further discussion can be found in the Appendix).

This SPOG* ${ }^{*}$ selection criteria suite was also used in Alatalo et al. (2014b), who show that SPOGs* as a population are likely to be found in the green valley and have Wide-Field Infrared Survey Explorer (WISE; Wright et al. 2010) infrared colors consistent with a transitioning population. Given that galaxy color was not a part of the criteria used to identify SPOGs*, this result is reassuring, in that, by using the SPOGs* criteria, we have indeed identified a population that appears to be quenching.

Figure 6 presents $30^{\prime \prime} \mathrm{g}$ ri SDSS cut-outs of the first 100 of 1067 SPOGs $^{*}$ in our sample ${ }^{14}$, and Table 2 presents the SPOG identifications, redshifts, $u \mathrm{~g} r i z$ de-reddened magnitudes from SDSS DR9 (Ahn et. al 2012), emission line fluxes from OSSY (Oh et al. 2011), $M_{r}$, $i$-band determined masses, and links to the SDSS explorer page. The catalog of SPOGs* is available through the NASA/IPAC Extragalactic Database (NED) ${ }^{15}$, this journal article, and the SPOGs website.

\footnotetext{
14 All thumbnails can be accessed through the online material, as well as via http://www.spogs.org.

15 http://ned.ipac.caltech.edu/
} 

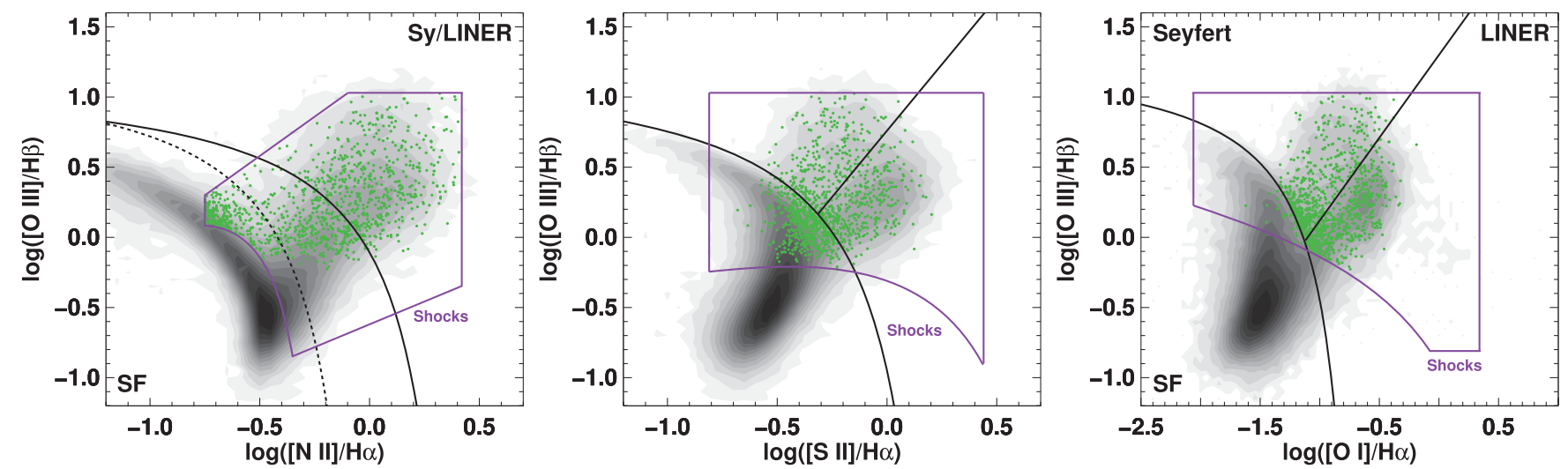

Figure 4. The line diagnostic diagrams (Baldwin et al. 1981; Veilleux \& Osterbrock 1987) for [O III] $/ \mathrm{H} \beta$ vs. [N II] $/ \mathrm{H} \alpha$ (left), [S II]/H $\alpha$ (center), and [O I] $/ \mathrm{H} \alpha$ (right) are overlaid with the ELG sample (grayscale) and SPOGs* (green points). Labels on $[\mathrm{N} \mathrm{II]} / \mathrm{H} \alpha$ and $[\mathrm{O}$ I] $/ \mathrm{H} \alpha$ label the locations of different ionizing mechanisms from Kewley et al. (2006) demarcated by black lines (the dashed line in the [N II]/H $\alpha$ plot is from Kauffmann et al. 2003). The composite region is shown between the dashed and solid black line on the $[\mathrm{N} \mathrm{II]} / \mathrm{H} \alpha$ diagram. Shock boundaries are overlaid in purple. SPOGs* populate a large fraction of the theoretically possible locations of shocks.

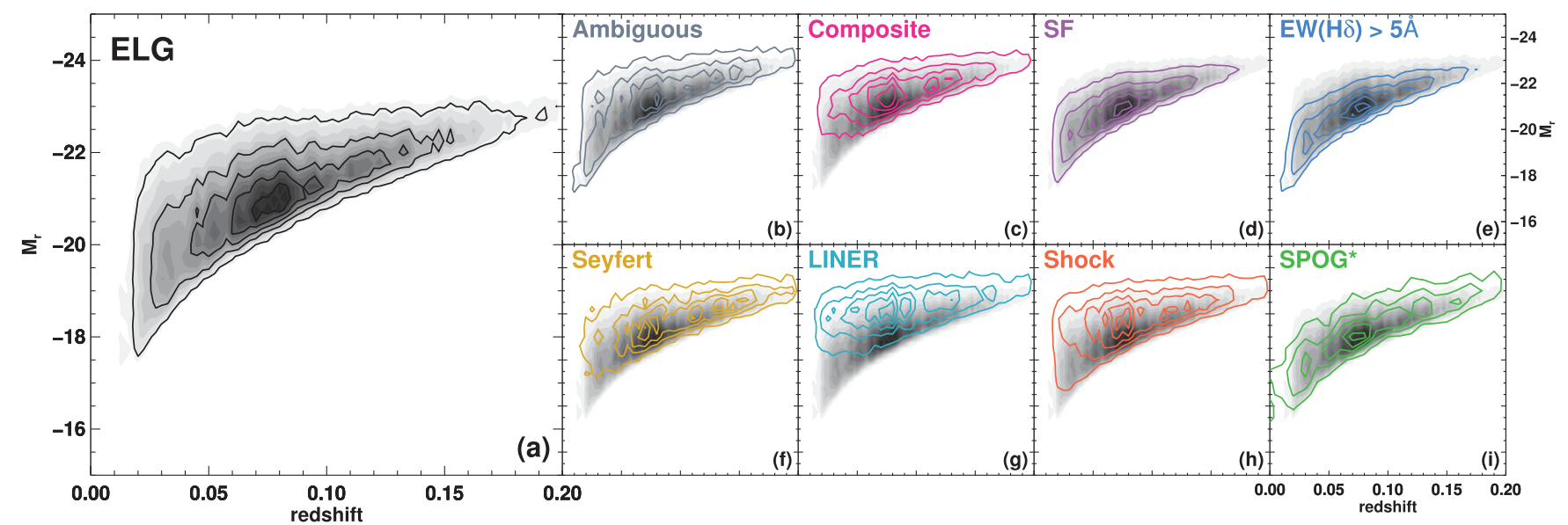

Figure 5. SDSS absolute $r$-band magnitude vs. redshift for the ELG (a; black, grayscale) overplotted with the distribution of objects that are spectrally classified as ambiguous (b: gray), composite (c: pink), SF (d: purple), those meeting the EW(H $\delta$ ) > $5 \AA$ criterion (e: blue), Seyferts (f: yellow), LINERs (g: turquoise), objects within the shock boundaries (h: red), and SPOGs* (i: green). Contours for all subsamples are in increments of 10\% from the maximum in each distribution. All objects are well represented in redshift space. Composites, Seyferts, and LINERs tend to have large $M_{r}$ and make up a higher fraction of the ELG at the higher end of our redshift range $(z=0.2)$. SPOGs ${ }^{*}$ typically have lower $M_{r}$, particularly at low $z$.

\section{DISCUSSION}

\subsection{Investigating Ionization Mechanisms of SPOGs*}

As discussed in Section 2.4, there are many mechanisms capable of creating emission line ratios consistent with shocks, such as SF, AGN activity, and photoionization from postasymptotic giant branch (post-AGB) stars. In Section 2.5, we identified a subset of galaxies with narrow-line diagnostics within the shock boundaries, but indicative of SF and thus mitigate contamination by omitting these systems from SPOGs*. For the remaining SPOGs*, we examine the likely ionization mechanisms and consider future observations to distinguish between them.

If the ionized gas originates from post-AGB stars, the narrow line emission (including the $[\mathrm{O} \mathrm{III}]$ ) should correlate with the stellar mass (Capetti \& Baldi 2011; Yan \& Blanton 2012). Figure 7 shows the distributions of [O $\mathrm{III}]$ versus SDSS $i$-band fiber magnitudes of the ELG and each of our subsamples. ELGs show two distinct branches, one shows a clear correlation between [O III] and $i$-band flux, and the other branch at fainter $i$-band magnitude is uncorrelated with [O III]
LINERs show the strongest relationship between $i$-band and [O III], consistent with the hypothesis that LINER emission mainly originates in post-AGB stars in massive, early-type systems (Yan et al. 2006; also confirmed by the red colors in Figure 8). This relationship is also present in the ambiguous subsample, composites, and shocks, but is weak or absent from the SPOGs* distribution. SPOGs* look more like SF or Seyferts than LINERs or shocks, consistent with the idea that the ionized gas in SPOGs* are not primarily excited by post-AGB stars.

It is likely that a non-negligible number of SPOGs* contain AGNs (consistent with what is found in NGC 1266; Alatalo et al. 2011; Davis et al. 2012), but it is unclear the total fraction of SPOGs* in which an AGN photoionization is the dominant mechanism exciting the ionized gas. The fact that a minority $(18 \%)$ of SPOGs* exhibit Seyfert-like line ratios means that our contamination from moderate (Seyfert) luminosity AGNs does not overwhelm the SPOGs* sample. Low-luminosity AGNs (LLAGNs) could also mimic the shock emission of the SPOGs* sample. LLAGNs are able to exhibit both LINERlike and ambiguous emission line ratios, though the emission 


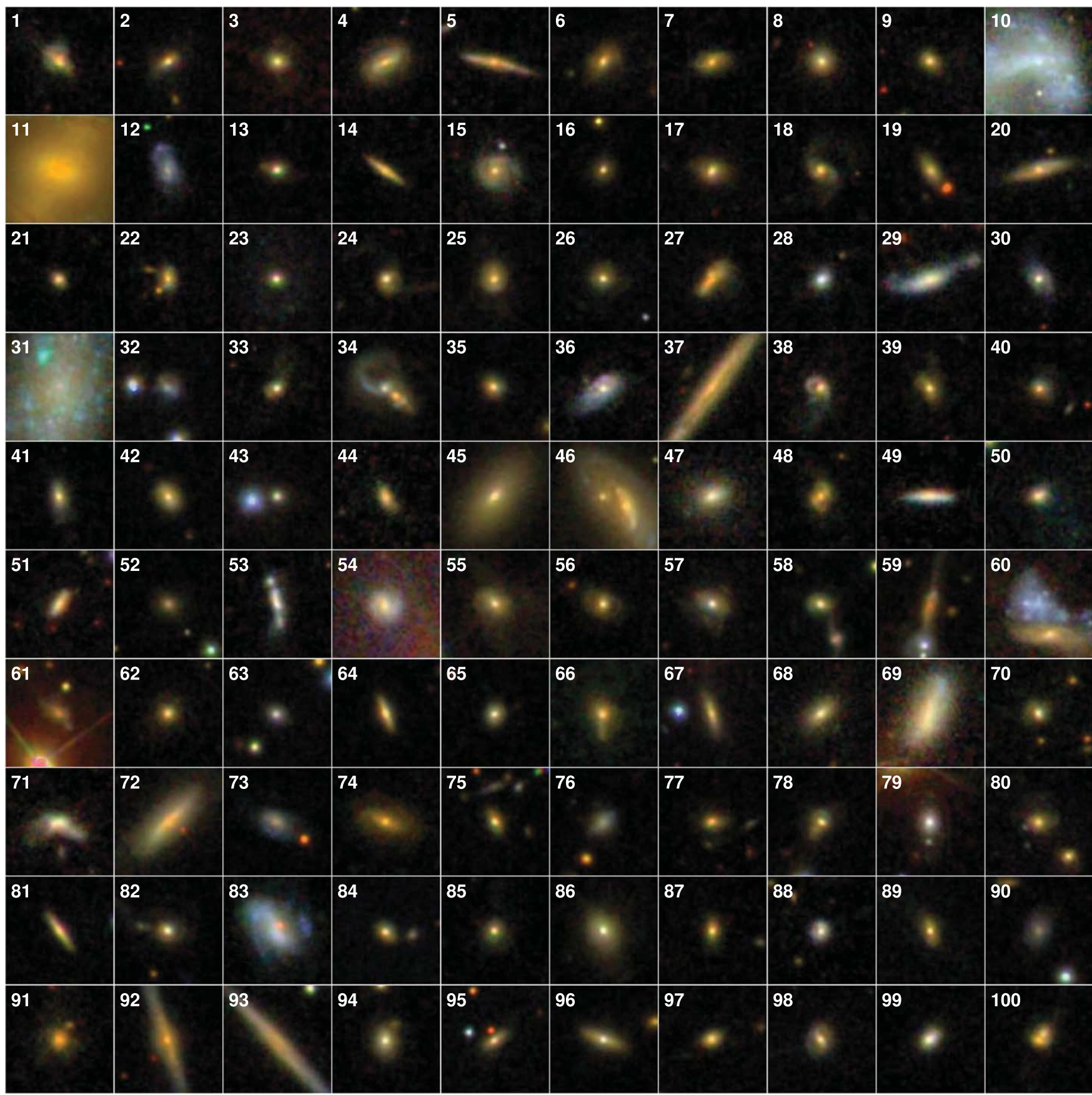

Figure 6. SDSS $g$ ri images of the first 100 SPOGs*, laid out in order of right ascension. Each thumbnail was pulled from SDSS DR12 (Alam et al. 2015), with fields of view of $30^{\prime \prime}$ in all cases. The $3^{\prime \prime}$ SDSS fiber is located at the center of the image. All other thumbnails are available in the online material, and are representative of the morphologies seen throughout the rest of the survey. Thumbnails of all SPOGs can be found at http://www.spogs.org. (An extended version of this figure is available.)

would be isolated to the nucleus. LLAGNs therefore could be a contamination source, especially at low- $z$ where the $3^{\prime \prime}$ SDSS fiber is only able to probe the ionization environment in the nucleus of the galaxy. As the SDSS fiber subtends larger area on the galaxy, it probes larger physical regions, possibly finding shock-like emission, which is usually extended. A more detailed discussion of how the line diagnostics and SPOG* detections change over redshift and mass can be found in the Appendix.
Integral Field Spectroscopy (IFS) shows promise at identifying shocks. A recent IFS study of NGC 7130 suggested that the two $[\mathrm{N} \mathrm{II}] / \mathrm{H} \alpha$ peaks (which lacks coincident peaks of emission in the near and mid-infrared) are energized by shocks tracing an outflowing wind, possibly by an AGN or SF (Davies et al. 2014). IFS studies of extended LINER-like emission revealed the presence of extended shock excitation in nearby major galaxy mergers (Monreal-Ibero et al. 2006, 2010; Rich et al. 2010, 2011, 2014, 2015). Extended shocks are often 


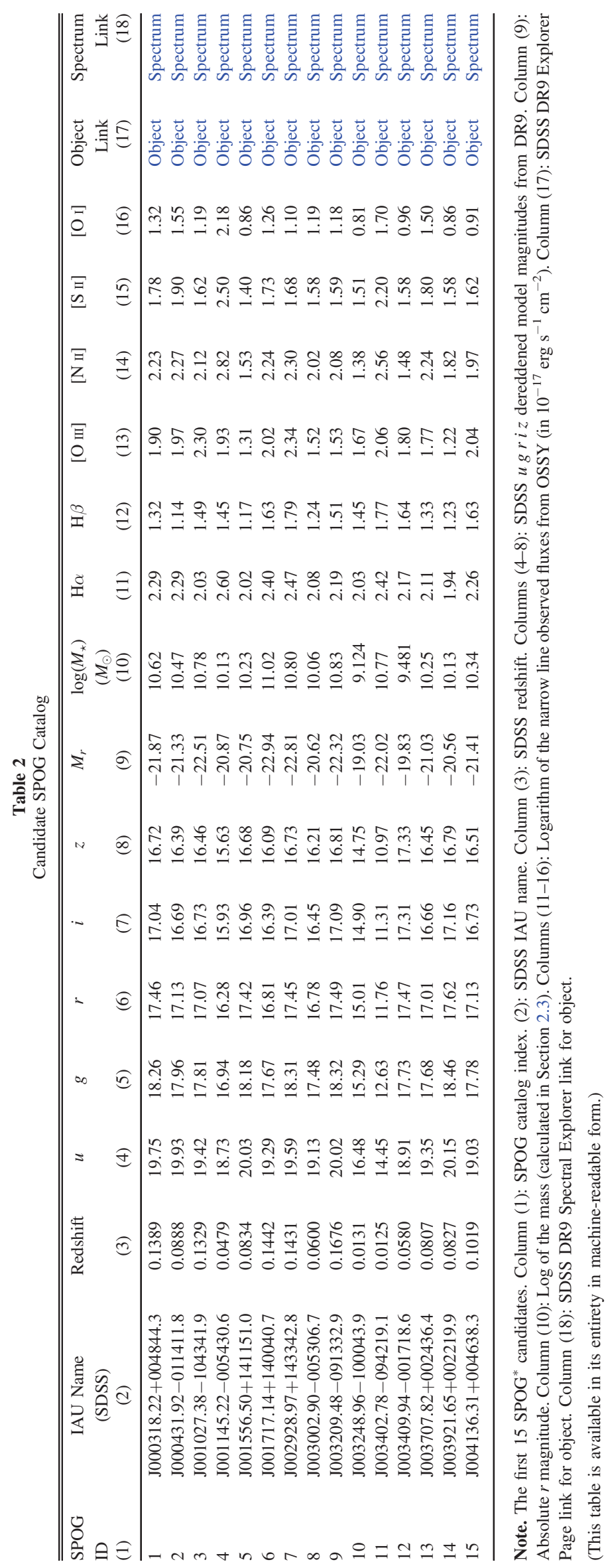




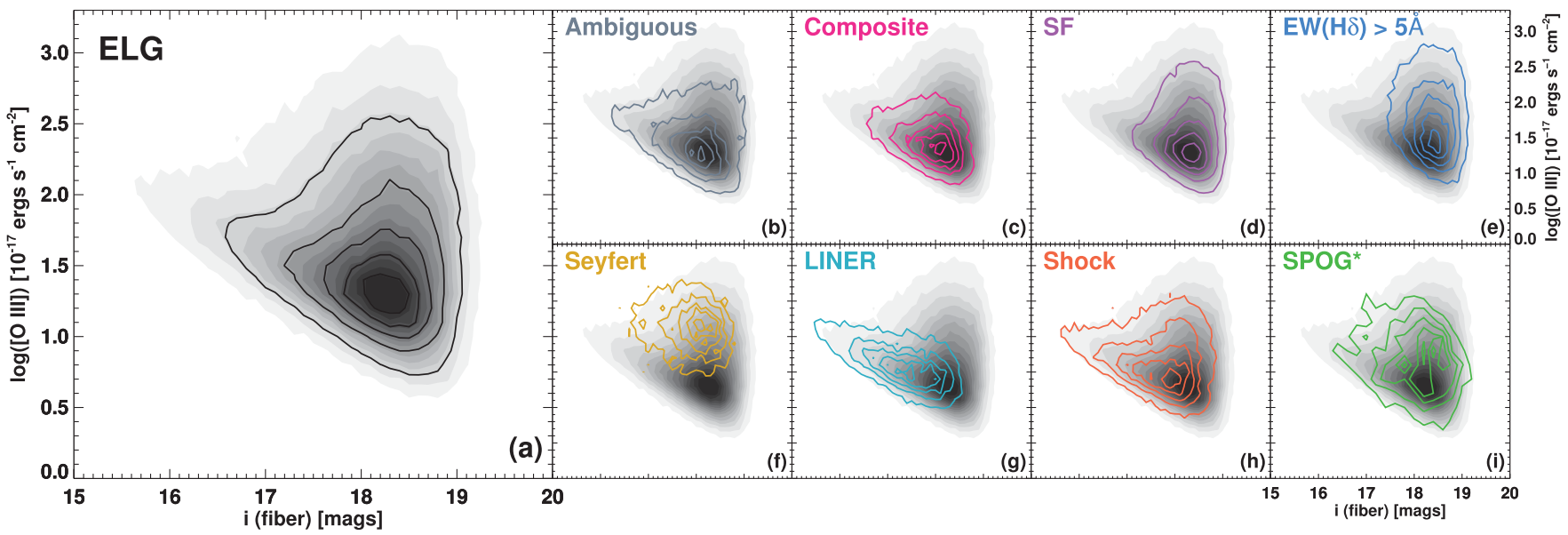

Figure 7. [O III] flux vs. apparent $i$-band fiber magnitude for the ELG (a; black, also grayscale) overplotted with the distribution of objects that are spectrally classifiied as ambiguous (b: gray), composite (c: pink), SF (d: purple), those meeting the EW(H $\delta)>5 \AA$ criterion (e: blue), Seyferts (f: yellow), LINERs (g: turquoise), objects within the shock boundaries (h: red), and SPOGs* (t: green). Contours for all subsamples are in increments of $10 \%$ of the maximum in each distribution. The ELG seems to show two branches, with one in which [O III] emission excited proportional to the stellar mass, most apparent in LINERs, but also present in ambiguous classifications, composite objects and shocks.

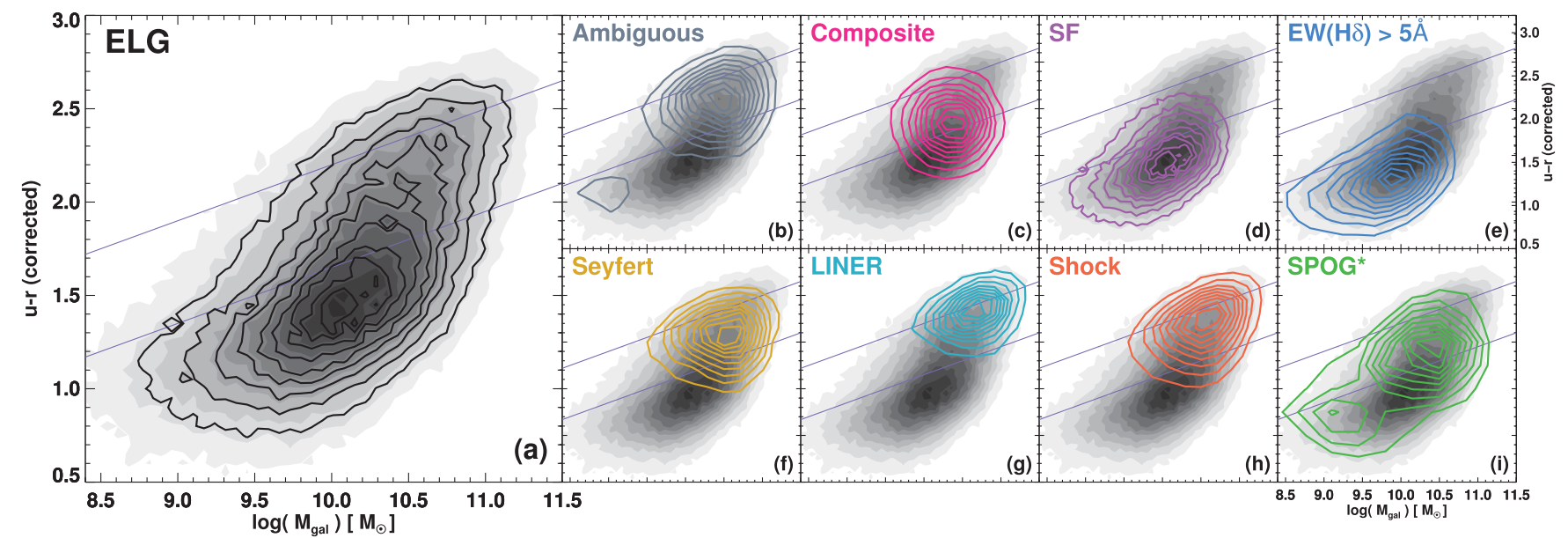

Figure 8. Corrected $u-r$ color-mass diagram (see Section 2.3) calculated for the ELG sample (a; black, also grayscale) overlaid with distributions of objects spectrally classified as ambiguous (b: gray), composite (c: pink), SF (d: purple), meeting the EW(H $\delta$ ) $>5 \AA$ criterion (e: blue), Seyferts (f: yellow), LINERs (g: turquoise), objects within the shock boundaries (h: red) and SPOGs* (i: green). Contours for all subsamples are $10 \%$ of the maximum. The indigo lines represent the green valley defined by Schawinski et al. (2014).

indicative of galactic wind-driven feedback and are easily discernible with IFS, but may appear as simple AGN or composite-like emission in spatially unresolved spectroscopy (Rich et al. 2014).

Large IFS studies such as the Calar Alto Legacy Integral Field Area, the Sydney AAO Multi-Object Integral Field Spectrograph, and the Mapping Nearby Galaxies at APO (MaNGA) surveys are observing thousands of galaxies with IFS (Croom et al. 2012; Sánchez et al. 2012; Bundy et al. 2015). These studies have already revealed the presence of shocks and winds in more "normal" nearby galaxies (e.g., Fogarty et al. 2012; Kehrig et al. 2012; Ho et al. 2014, 2016). Studying SPOGs* with IFS will not only reveal interesting substructure and 2D kinematics of the emission present but will be able to remove a possible contaminant to the sample: AGNs. SPOGs* with emission that is excited by shocks will appear extended when observed with an IFS, while a LLAGN will manifest as a point source consistent with the nucleus.
SPOGs* have optical emission-lines consistent with shocks, but optical diagnostic ratios alone are degenerate with other processes. Near-IR emission lines such as [Fe II] and rovibrationally excited $\mathrm{H}_{2}$ are able to break this degeneracy. Shock excitation manifests itself in large [Fe II]1.64/Br $\gamma$ emission line ratios (Mouri et al. 2000) and strong $\mathrm{H}_{2}$ lines. Thus, $\left[\mathrm{Fe}\right.$ II] line detection in conjunction with the $\mathrm{H}_{2}$ lines will reveal differences in excitation and kinematics away from the nuclei (see Mouri et al. 2000). The two main excitation routes for ro-vibrational molecular hydrogen emission are UV florescence and collisions in hot gas in shocks (Shull \& Beckwith 1982; Black \& van Dishoeck 1987), and these can be distinguished by looking at the relative strength of the rovibrational line fluxes, 2-1S(1)/1-0S(1); typically 0.1 for $T=2000 \mathrm{~K}$ shocks, and $\sim 0.5$ in UV-pumped star-forming regions. Detection of multiple transitions of ro-vibrational $\mathrm{H}_{2}$ will be able to unambiguously identify the presence of shocks in SPOGs*, and if the spectra are taken using a longslit spectrograph or an IFS, extended shocks can be mapped, 
searching for emission similar to the extended winds seen in NGC 1266 (Alatalo et al. 2011) or high redshift quasars (Nesvadba et al. 2008). The first results of near-IR observations of ro-vibrationally excited $\mathrm{H}_{2}$ detections in SPOGs* will be presented in an upcoming paper (K. Alatalo et al. 2016, in preparation).

\subsection{Are SPOGs ${ }^{*}$ Entering or Leaving the "Green Valley"?}

Figure 8 presents a $u-r$ color-mass diagram for the ELG and SPOG* ${ }^{*}$ samples. The ELG catalog, shown in the large panel to the left and as the grayscale contours underlying the subsamples, primarily occupies the blue cloud. Contours of each of the subsamples described above are overplotted in the small panels. Composites tend to be slightly bluer than Seyferts, and are much more likely to be found in the green valley than pure star-forming objects. Objects classified as ambiguous contain two optical color peaks: one similar to Seyferts that might be galaxies lying along the Seyfert-LINER mixing line (Rich et al. 2014), and one at low-mass lowredshift that might be low metallicity dwarfs (discussed in the Appendix). Objects that fall within shock boundaries are most likely to be near the red sequence, similar to LINERs. $\mathrm{EW}(\mathrm{H} \delta)>5 \AA$ objects have the bluest peak of all subsamples represented here, which is probably due to their likelihood to harbor narrow-line ratios typical of star forming regions, and their high incidence of A-stars, which peak in blue optical light. SPOGs ${ }^{*}$ have a high fraction of objects in the green valley, but with a bluer peak than composites or Seyferts, thus they likely have a larger population of young stars. SPOGs* (according to their narrow-line diagnostics) are not actively forming stars, and thus are not as blue as the star-forming and $\mathrm{EW}(\mathrm{H} \delta)>5 \AA$ A objects.

The location of SPOGs* on the blue edge of the green valley (Figure 8(i)) indicates that they might be recent arrivals to the green valley. As described in Alatalo et al. (2014b), SPOGs* are also found to have WISE colors consistent with entering the transition zone rather than leaving. Figure 9 compares the $u-r$ colors of SPOGs ${ }^{*}$ to the colors ${ }^{16}$ of OSSY galaxies that pass the continuum $\mathrm{S} / \mathrm{N}$ cuts used for the ELG sample (Section 2.1), but with an additional " $\mathrm{E}+\mathrm{A}$ " criterion of Goto $(2007)^{17}$ applied. The OSSY E+A objects on average have green colors, with a wing extending to the red sequence. The fact that the SPOGs* peak is bluer than the E+A peak (objects that are known to be transitioning), but still consistent with the optical green valley is a promising sign that we have identified the transforming population that we are searching for. When studied in detail, SPOGs* might be able to elucidate the behaviors of galaxies at earlier stages of transition, especially given that green valley and poststarburst galaxies appear to show signs of having morphologically transitioned (Yang et al. 2004, 2008; Wong et al. 2012). In order to detail the evolution of SPOGs*, analyses of the UV properties, spectral energy distributions, and stellar population synthesis modeling are underway (L. Lanz et al. 2016, in preparation).

It is also possible that SPOGs* are not LTGs entering the green valley from the blue sequence, but rather ETGs that have been replenished in molecular gas and entered the green valley

\footnotetext{
${ }^{16} k$-corrected, dereddened, extinction corrected colors derived from the SDSS model mags.

${ }^{17} \mathrm{H} \delta$ absorption: $\mathrm{EW}(\mathrm{H} \delta)>5 \AA$, absense of emission: $\mathrm{EW}([\mathrm{O}$ I $])<2.5 \AA$, $\mathrm{EW}(\mathrm{H} \alpha)<3 \AA$.
}

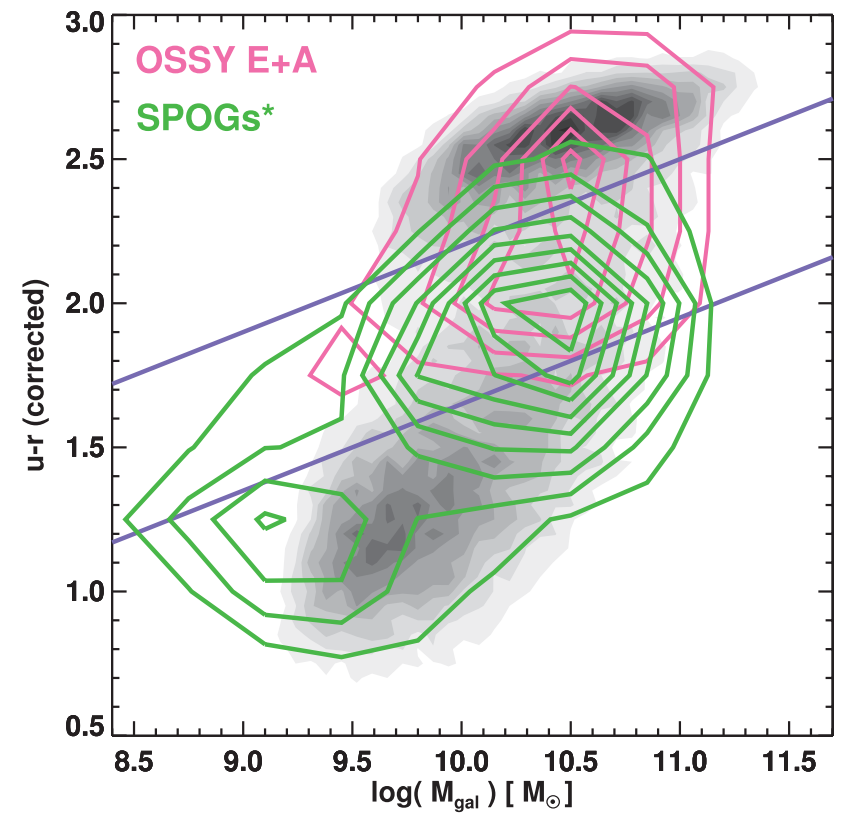

Figure 9. The optical color-mass distributions for the Galaxy Zoo sample (Schawinski et al. 2014; grayscale), compared to SPOGs* (green contours) and "E+A" (criteria from Goto 2007) galaxies from the OSSY catalog that meet the continuum $\mathrm{S} / \mathrm{N}$ requirements of the ELG catalog (pink contours). The OSSY "E+A" galaxies are found in the optical green valley (as expected; Dressler \& Gunn 1983) with a wing in the red sequence. Poststarburst galaxies are also (on average) more massive than SPOGs* (though it is likely this is due to the signal to noise cuts applied to the OSSY E $+\mathrm{A}$ sample). The green valley definition from Schawinski et al. (2014) is bounded by indigo lines. The SPOG* contours confirm that SPOGs* on average are bluer than poststarburst galaxies, including in comparable mass bins.

from the red sequence. Dressler et al. (2013) presented a large sample of galaxies inside and outside clusters between $0.3<z<0.5$. The authors found that galaxies with poststarburst stellar signatures had a mass distribution consistent with that of the ETG, whereas the mass distribution of starbursting galaxies was consistent with the mass distribution of LTGs, independent of environment. The authors went on to suggest that this mismatch argued for different origins for poststarburst and starburst galaxies, with the majority of poststarburst galaxies being replenished early-types. Thus, as with the poststarburst galaxies from Dressler et al. (2013), it is possible that the origin of SPOGs* is not quenching LTGs, but rather ETGs with replenished gas reservoirs. In this case, the difference between SPOGs* and poststarbursts could be either a larger accreted gas reservoir or objects caught earlier in the process of replenishing. A detailed mass distribution, similar to what was done in Dressler et al. (2013) will be required to determine if replenished early-types are a major contributor to the SPOG phase.

\subsection{The SPOG Lifetime}

Poststarburst stellar populations occur when current SF terminates abruptly $(<100 \mathrm{Myr})$ and remains dormant for several 100 Myr. Typical spectra display Balmer jumps and high order Balmer absorption lines common of A-type stars. The detectability of these spectral signatures is set to first order by the main sequence lifetimes of A-stars ( 1 Gyr; Falkenberg et al. 2009), but in most cases is only detectable for 0.1-0.3 Gyr (Snyder et al. 2011). Thus, these galaxies are thought to be in transition between actively star-forming LTGs and passive 
ETGs observable on a timescale of $100 \mathrm{~s}$ of Myr. This is consistent with the notion that ETGs move rapidly across the green valley from blue cloud to red sequence in several 100 Myr to $\sim 1 \mathrm{Gyr}$, after their morphologies are transformed from disk to spheroid and SF is quenched rapidly (Schawinski et al. 2014).

The 1067 SPOG candidates represent $0.2 \%$ of the OSSY sample that meet the continuum $\mathrm{S} / \mathrm{N}$ requirements $(591,627$ objects), and $0.7 \%$ of the ELG sample. Catalogs of poststarburst galaxies usually number in the several hundreds and tend to account for $0.2 \%$ of galaxies (Zabludoff et al. 1996; Quintero et al. 2004; Goto 2007; Pattarakijwanich et al. 2014). There were $694(0.1 \%)$ objects in the OSSY continuum sample that met the $\mathrm{E}+\mathrm{A}$ criteria of Goto (2007), within a factor of two of the number of SPOGs* we identify. In both the cases of OSSY E $+\mathrm{A}$ and SPOG samples, selection biases remain. In the case of SPOGs*, our line intensity criterion (the significant detection of all diagnostic lines) likely removes SPOGs* from the survey, so $0.2 \%$ is a lower limit. Similarly, poststarburst galaxies include only objects with weak or absent nebular line ([O II] or $\mathrm{H} \alpha$ ) emission, despite there being multiple non-star-forming mechanisms capable of exciting them. In this case, because the SPOG and "E $+\mathrm{A}$ " criterion sample a nearly mutually exclusive parameter space, it is possible that we can add SPOGs* to the overall poststarburst/post-transition galaxy sample. Our new method of selecting post-transition galaxies, while allowing for them to harbor powerful sources of ionization from AGN, LINERs, and/or shocks, provides a sizable, heretofore neglected, fraction of the total transitioning population.

If we assume that the fraction of SPOGs* among the OSSY continuum sample roughly represents the fraction of SPOGs* in the local universe, we can derive a rough estimate of the amount of time that galaxies spend in a SPOG phase. Assuming that all galaxies go through a single SPOG phase over a Hubble time, we find an average SPOG timescale of $t_{\mathrm{SPOG}} \approx 20 \mathrm{Myr}$. Given the selection biases in place (including the requirement of finding strong [O I] emission), this is likely a lower limit on the timescale. Assuming $t_{\text {SPOG }}$ represents a lifetime of a SPOG episode, the fact that it roughly agrees with the dissipation time of shocks $(\sim 10-100 \mathrm{Myr}$; Guillard et al 2009; Lesaffre et al. 2013) suggests that the SPOG lifetime might be driven by the timescale that shocks are illuminating the galaxy, rather than the timescale over which intermediate-aged stars can be detected. This is likely an additional reason that the poststarburst lifetime does not match the timescale of A-stars: that non-star forming ionization mechanisms might be producing nebular line emission in the galaxy after quenching, causing the " $\mathrm{E}+\mathrm{A}$ " survey to miss transitioning objects.

In the context of merger driven evolutionary scenarios, rapid bursts of SF ( $\tau_{\text {quench }} \lesssim 100 \mathrm{Myr}$ ) happen early in the merger and coincide with the close passages of the two nuclei (Di Matteo et al. 2005; Hopkins et al. 2008; Van Wassenhove et al. 2012; Stickley \& Canalizo 2014). While the merger and its signatures can last 1-2 Gyr (Di Matteo et al. 2005), each successive pass of the nuclei occurs on timescales of several $100 \mathrm{Myr}$ (Stickley \& Canalizo 2014), and final coalescence of the nuclei is quick ( $\lesssim 100 \mathrm{Myr}$; Lanz et al. 2014). Mergers are effective at triggering shocks (Rich et al. 2011, 2014, 2015; Soto et al. 2012; Inami et al. 2013); because the merger rate for the nearby universe (Darg et al. 2010; Lotz et al. 2011) agrees with the SPOG* fraction, it is possible that mergers undergo a
SPOG phase. Given that the starburst timescale within mergers is longer than the shock dissipation time, signs of the SPOG phase in mergers are likely overpowered by the remnant SF (Rich et al. 2011). IFS studies can delineate spatially between shock-dominated, and SF dominated regions (Rich et al. 2015), and might provide a path forward to identifying whether major mergers transit through a SPOG phase.

Shocks are also able to increase the molecular gas depletion time, and therefore the SF quenching time, in galaxies, by inhibiting SF. Shocks introduce excess kinetic energy that counteracts the gravitational instabilities within the gas that regulates SF, leading to longer molecular gas depletion times. This phenomenon has been seen in AGN outflow hosts (Aalto et al. 2015; Alatalo et al. 2015b), radio galaxies (Guillard et al. 2015; Lanz et al. 2015), and warm $\mathrm{H}_{2}$-bright Hickson Compact Group galaxies (Guillard et al. 2012; Alatalo et al. 2014a, 2015a). Shock-driven turbulence produced as a result of cyclical AGN episodes is one of the mechanisms capable of regulating SF by rendering molecular gas infertile (Alatalo et al. 2014c), possibly extending the lifetime of the SPOG phase. If shocks are able to regulate SF in SPOGs, this suggests that the SPOG phase could include multiple shortduration SPOG episodes over a longer timescale (such as the A-star lifetime). A similar effect has been suggested for AGNs (Schawinski et al. 2015).

Regardless of the demographics of SPOGs, in order to detect poststarburst spectral features, the SF quenching timescale must be rapid. It is unclear whether a galaxy undergoing a SPOG phase is transforming via a quietly quenching mechanism (such as a minor merger or cosmic gas starvation), a major merger, cluster encroachment, group $\lesssim 100$ Myr timescales. An in-depth study of the environments and morphologies, including deep observations looking for stellar or $\mathrm{H}_{\mathrm{I}}$ tidal streams would aid in constraining the fraction of each of these types of transformations that undergoes a detectable SPOG phase.

\subsection{Interstellar Na I D in SPOGs* : Evidence of Winds?}

The neutral sodium doublet ( $\mathrm{Na}$ I $\mathrm{D} \lambda 5890,5896)$ is a stellar absorption feature in the atmospheres of cool stars (Bruzual \& Charlot 2003; Vazdekis et al. 2010) and a well-known ISM absorption line. In the past few decades, interstellar $\mathrm{Na}$ I D has been detected in galactic winds in both starburst systems (Heckman et al. 2000; Rupke et al. 2005; Martin 2006; Chen et al. 2010; Sarzi et al. 2016) and AGN-driven outflow systems (Forster et al. 1995; Krug et al. 2010; Davis et al. 2012; Rupke \& Veilleux 2015).

Figure 10 shows the $\mathrm{Na}$ I D versus $\mathrm{Mg}$ b absorption in ELG sample and subsamples. There is a tight relationship between $\mathrm{Mg} \mathrm{b}$ and $\mathrm{NaID}$ absorption throughout the ELG and most subsamples, consistent with the majority of the NaID absorption having a stellar origin. The $\mathrm{Na}$ I D deficit at small $\mathrm{Mg} b$ that appears in the star-forming, $\mathrm{EW}(\mathrm{H} \delta)>5 \AA$, and ELG samples is likely caused by infilling of both the $\mathrm{Na}$ I and nearby $\mathrm{He}$ I emission lines, which is strongest in young and metal-poor stellar populations.

An empirical relationship between $\mathrm{Mgb}$ and $\mathrm{Na}$ I D in the ELG can be derived, resulting in the following equation:

$$
\mathrm{EW}(\mathrm{Na} \text { I D })=0.685 * \mathrm{EW}(\mathrm{Mg} \mathrm{b})+0.8 .
$$

Most of the ELG subsamples trace the Na I D-Mg b relation of Equation (10) (shown as a dashed blue line on Figure 10(a)). SPOGs ${ }^{*}$ are the marked exception. The $\mathrm{Na}$ I D-Mg b relation in 


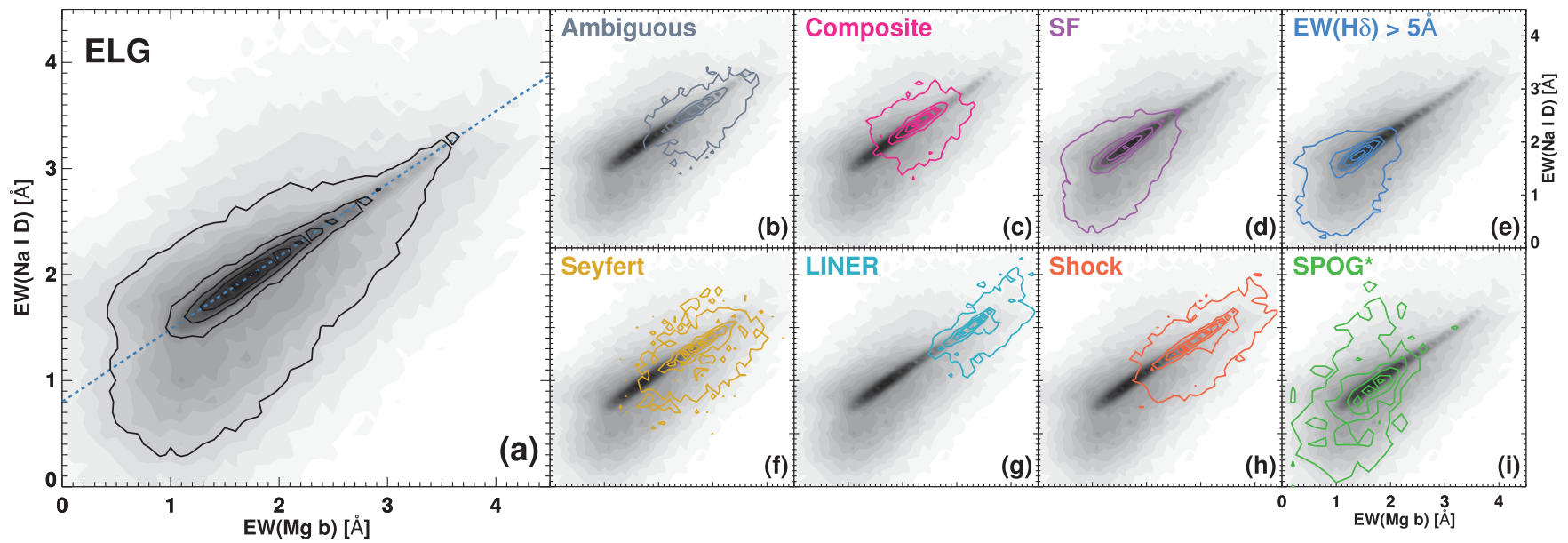

Figure 10. Na I D absorption is compared to Mg b for the ELG sample (a; black, underlying grayscale), with panels for ambiguous objects (b: gray), composites (c: pink), SF (d: purple), EW(H $\delta)>5 \AA$ objects (e: blue), Seyferts (f: yellow), LINERs (g: turquoise), objects within the shock boundaries (h: red), and SPOGs* (i: green). Contours for all subsamples are in increments of $10 \%$ from the maximum in each distribution. In general, the Mg b and $\mathrm{Na}$ I D absorption trace stellar population age (Vazdekis et al. 2010), as is seen in the underlying parent sample. We see the general trend that LINERs contain the oldest stellar populations of the ELG sample, and EW(H $\delta)>5 \AA$ objects the youngest. SPOGs* are the only population that has a non-negligible representation of galaxies with much larger Na I D widths than Mg b, consistent with neutral winds (Rupke et al. 2005; Murray et al. 2007; Jeong et al. 2013).
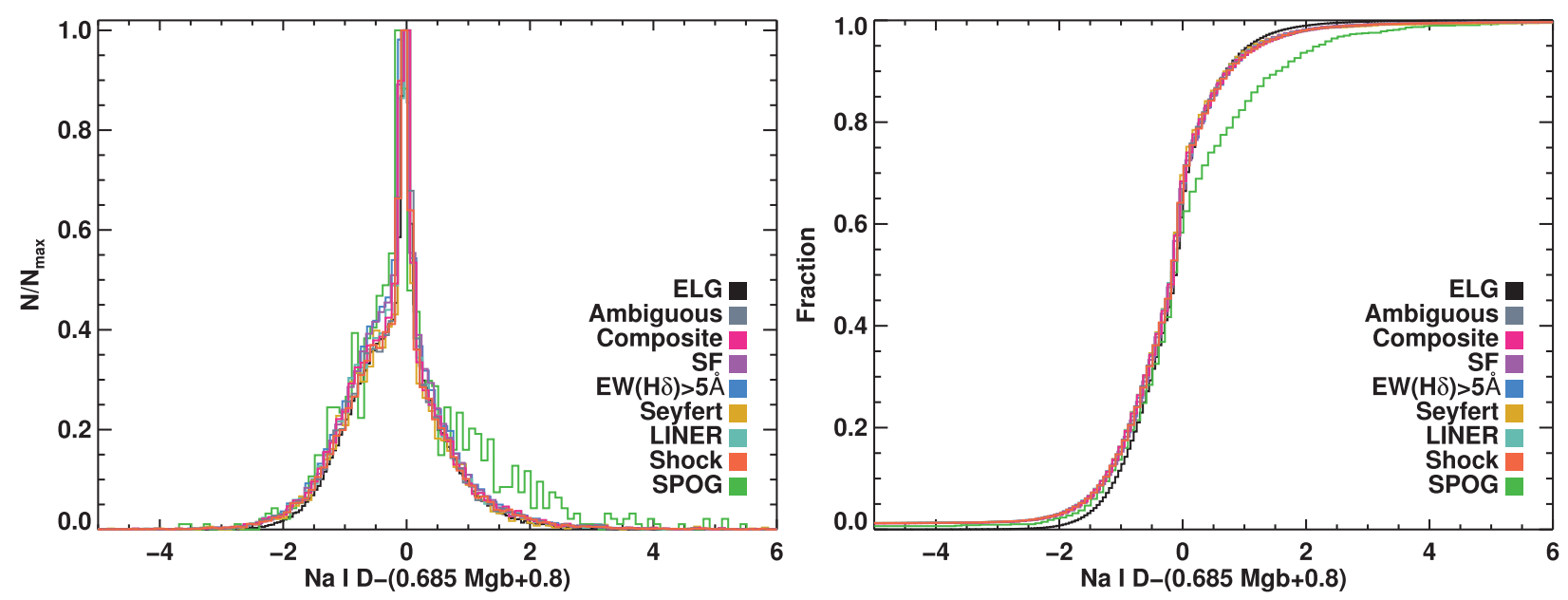

Figure 11. The probability distribution function (left) and the cumulative distribution function (right) for the relative Na I D vs. Mg b in our parent sample (ELG) and subsamples, collapsed along the $\mathrm{EW}(\mathrm{Na} \mathrm{D})=0.685^{*} \mathrm{EW}(\mathrm{Mg} \mathrm{b})+0.8$ line shown in Figure 10 . SPOGs* show a clear departure from all other distributions, with a larger fraction of objects that are $\mathrm{Na}$ I D-strong for their $\mathrm{Mg} \mathrm{b}(23 \%$ are beyond 1 standard deviation off of the ELG-derived relation), indicative of Na I D winds (Murray et al. 2007).

SPOGs* most closely resembles the relation in $\mathrm{EW}(\mathrm{H} \delta)>5 \AA$ objects, but only at the small $\mathrm{Na}$ I D end. SPOGs* have a substantial (and unique) tail into strong Na I D absorption (compared to $\mathrm{Mgb}$ ). Figure 11 shows the probability distribution function (PDF; left) and the cumulative distribution function (CDF; right) of the Na I D-Mg b relation, collapsed along the line described by Equation (10). While most subsamples follow the same distribution around Equation (10), SPOGs* show a deviation, evident in both the PDF and the CDF. A non-parametric Kolmogorov-Smirnov test ${ }^{18}$ was run to compare SPOGs ${ }^{*}$ to the other distributions, with the null hypothesis being ruled out with a confidence $p \ll 0.001$. In detail, 245 (23\%) SPOGs* are at least 1 standard deviation $(1 \sigma)$ above Equation (10), $120(11 \%)$ at least $2 \sigma$, and $56(5 \%)$

18 http://idlastro.gsfc.nasa.gov/ftp/pro/math/kstwo.pro
SPOGs $^{*}$ exceed Equation (10) by at least $3 \sigma$, confirming that a substantial population of SPOGs* show significant Na ID enhancement above what is expected from stellar contributions based on $\mathrm{Mg} \mathrm{b}$ absorption.

Enhanced $\mathrm{NaID}$ absorption is also observed due to a varying initial mass function (IMF) for the stars (Jeong et al. 2013; McConnell et al. 2015), but this has only been seen in the most massive, oldest systems. SPOGs* span a large range of masses, thus we do not believe that a varying IMF is the cause for the enhanced $\mathrm{Na}$ I D. Another possibility is that there is likely a nonstellar contribution to the $\mathrm{Na}$ I D line. The presence of interstellar NaID absorption among the SPOG* sample would suggest the presence of neutral winds, driving a non-negligible amount of ISM out of the system, thus aiding in the galaxy's transition.

Enhanced $\mathrm{Na}$ ID absorption has also been shown to be a signature of neutral winds (Rupke et al. 2005). It is possible 
that other subsamples may also host winds (such as the starforming subsample; Sarzi et al. 2016). Given the enhancement in $\mathrm{Na}$ I $\mathrm{D}$ absorption over Mg b, SPOGs* may have proportionally more interstellar neutral material as a population than the other subsamples. Additionally, given the masses and A-star populations seen in SPOGs*, the enhanced $\mathrm{Na}$ I D is much more likely to be due to interstellar winds. Na I D winds found in AGN-driven molecular outflows, such as Markarian 231 (Rupke \& Veilleux 2011) and NGC 1266 (Davis et al. 2012) were concentrated in the center, and traced the molecular outflow from the AGN. The velocity dynamics in both these systems made it clear that this absorption is due to the AGNdriven neutral wind. Given that the discovery of NGC 1266 was the catalyst for the SPOG survey, finding that SPOGs* as a population also have $\mathrm{Na}$ I D absorption enhanced beyond what standard stellar population models predict is promising, and is a sign that our selection criteria have indeed produced the special population of transitioning galaxies that we were looking for.

This shows that SPOGs* satisfying our $\mathrm{EW}(\mathrm{H} \delta)>5 \AA$ and shock criteria are not a random subsample of ELGs; they have distinct $\mathrm{Na}$ I D behavior, showing a large wing with enhanced $\mathrm{Na}$ I D absorption. In starbursts, these neutral winds tend to be attributed to stellar feedback, but given that the SPOGs* selection actively excludes $\mathrm{SF}$, the enhanced $\mathrm{Na}$ I D absorption is likely from another source, such as AGN feedback. One potential caveat is that a NaID enhancement alone is not sufficient to confirm the presence of neutral winds, as enhanced $\mathrm{Na}$ I D can also come from tidal debris sitting in front of the stars. Thus, an in-depth study of the kinematics and extent of the $\mathrm{Na}$ I D absorption is necessary to determine the mass output and to confirm whether the NaID in SPOGs* is due to interstellar winds. A full investigation of the $\mathrm{Na}$ I $\mathrm{D}$ properties of SPOGs* will be presented in a future paper.

\subsection{Radio Properties of SPOGs*}

Feedback via the injection of turbulent energy driven by a low-power radio AGN was a key aspect of the discovery of NGC 1266 (Alatalo et al. 2015b). It is therefore of great interest to consider the potential influence of radio AGN feedback among SPOGs. We estimate the fraction of candidate "radioloud" AGN hosts in our sample of SPOGs using the Best \& Heckman (2012a) catalog of SDSS sources from DR7 with radio counterparts in the NRAO Very Large Array (VLA) Sky Survey (Condon et al. 1998) and the Faint Images of the Radio Sky at Twenty Centimeters (FIRST; Becker et al. 1995) Survey. The Best \& Heckman (2012a) catalog contains 18,286 radio-loud sources with flux densities measured at $1.4 \mathrm{GHz}$ that also have SDSS counterparts down to a limiting flux density of $5 \mathrm{mJy}$. Of the 1067 SPOGs in our sample, only $32(\approx 3.0 \%)$ are included in the Best \& Heckman (2012b) catalog of SDSS radio sources. To provide further insights into the population of faint radio sources with counterparts in our ELG catalog, we directly cross-matched this catalog with FIRST (with a flux limit of $1 \mathrm{mJy}$ ) using тоРсат (Taylor 2005). We followed the strategy of Ivezić et al. (2002), who argued that a search radius of 1 !' 5 yields the best compromise among completeness and contamination $(85 \%$ and $3 \%$, respectively) when crossmatching SDSS galaxies with FIRST. Using this search radius, the position of a radio source should lie within $3^{\prime \prime}$ of the optical position of a galaxy from our ELG catalog. Of the 159,387 objects in the ELG sample, 6351 (4\%) have FIRST matches. Table 3 provides information on the $1.4 \mathrm{GHz}$ detection rates for
Table 3

FIRST Detections

\begin{tabular}{lrrr}
\hline \hline Type & Number & Percent $^{\mathrm{a}}$ & $\left\langle\log \left(L_{1.4 \mathrm{GHz}}\right)\right\rangle^{\mathrm{b}}$ \\
\hline Ambiguous & 1000 & 5.9 & $22.574 \pm 0.019$ \\
Composite & 1127 & 7.9 & $22.364 \pm 0.014$ \\
SF & 2362 & 2.1 & $22.212 \pm 0.010$ \\
EW $(\mathrm{H} \delta)>5 \AA$ & 1136 & 2.4 & $22.342 \pm 0.014$ \\
Seyfert & 349 & 7.3 & $22.687 \pm 0.037$ \\
LINER & 1513 & 13.4 & $22.853 \pm 0.020$ \\
Shocks & 2625 & 8.7 & $22.731 \pm 0.014$ \\
SPOGs* & 143 & 13.4 & $22.823 \pm 0.047$ \\
ELG & 6351 & 4.0 & $22.450 \pm 0.008$ \\
\hline
\end{tabular}

Notes.

${ }^{\text {a }}$ Percent of specific subsamples with FIRST matches.

${ }^{\mathrm{b}}$ The mean $\log 1.4 \mathrm{GHz}$ luminosities with standard deviation of the mean.

each subsample of objects within the ELG catalog. Figure 12 shows the distributions of $1.4 \mathrm{GHz}$ radio luminosities within the ELG sample and line diagnostic subsamples. LINERs, Seyferts, shocks, and SPOGs* have more significant luminosities than the $\mathrm{SF}$ and $\mathrm{EW}(\mathrm{H} \delta)>5 \AA$ objects, given the shift seen in the radio luminosity distribution compared with the mean of the whole ELG sample (also shown in Table 3). Figure 13 shows the archival $1.4 \mathrm{GHz}$ luminosity dependence on redshift and mass for the ELG sample. Radio-detected objects span all redshifts and stellar masses. A detailed statistical analysis of the radio continuum properties of the ELG sample and SPOGS, in particular, one that addresses the inherent distance and galaxy mass biases inherent to fluxlimited surveys such as FIRST (e.g., Best et al. 2005 ), will be presented in a future study.

The subsamples with the highest radio detection rates are SPOGs* and LINERs (both 13\%), significantly exceeding the Seyfert classified objects $(7 \%)$. The high fraction of radiodetected LINERs is expected based on previous studies showing they are often hosted by massive galaxies with radio-loud AGNs (Owen \& Laing 1989). We speculate that the similarly high incidence of radio sources in SPOGs* could be a sign that these objects also host radio-loud AGNs, however, we emphasize that the detection of a weak radio source does not guarantee that an AGN present since SF may also produce radio emission at low levels (Condon 1992). In addition to synchrotron emission from AGNs and recent SF, shocks are known to produce radio continuum emission as well (Lisenfeld \& Völk 2010). Thus, determining which mechanism dominates the energetic output of a galaxy is challenging. Studies investigating the radio- $22 \mu \mathrm{m}$ correlation (e.g., Appleton et al. 2004) in the ELG sample to help identify objects in which AGN emission dominates at radio frequencies will be presented in future work.

\subsection{Potential Catalysts of the SPOG Phase}

Radio jets could explain many observational properties of SPOGs: their short timescales, their lack of ongoing SF, and the fact that they harbor powerful sources of ionization. Jets from radio galaxies can inject significant turbulence (traceable via shocks) into a galaxy's ISM, thereby inhibiting its ability to form stars (Lanz et al. 2015). Strong radio galaxies have been found to be cyclical through the discovery of fossil radio shells (Schoenmakers et al. 2000), extending the radio bright timescale beyond the radio jet timescale $(\sim 10 \mathrm{Myr}$; Turner \& 


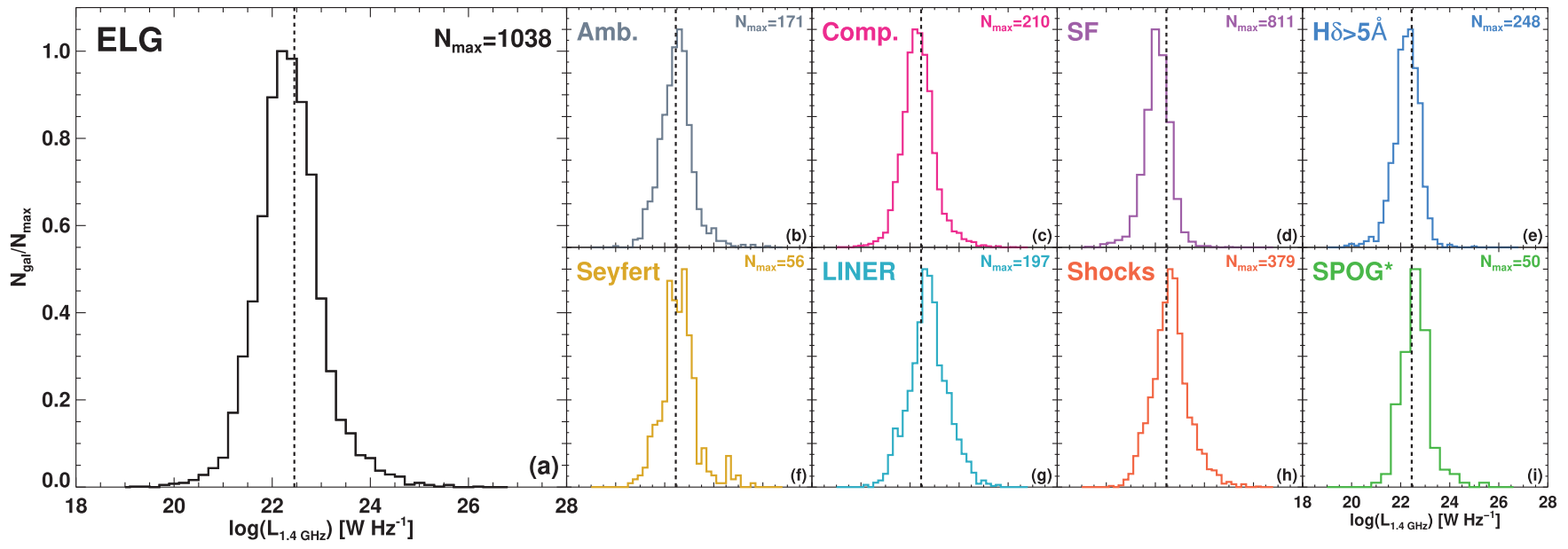

Figure 12. Radio luminosity histogram of FIRST-detected objects in the ELG sample (a; black), ambiguous objects (b: gray), composites (c: pink), SF (d: purple), $\mathrm{EW}(\mathrm{H} \delta)>5$ A objects (e: blue), Seyferts (f: yellow), LINERs (g: turquoise), objects within the shock boundaries (h: red), and SPOGs* (i: green). The dotted black line represents the mean radio luminosity of all ELG objects. $N_{\max }$ represents the number of objects in the most populated bin. The average $L_{1.4 \mathrm{GHz}}$ for SF and $\mathrm{EW}(\mathrm{H} \delta)>5 \AA$ objects is smaller than in LINERs, Seyferts, shocks, and SPOGs*.

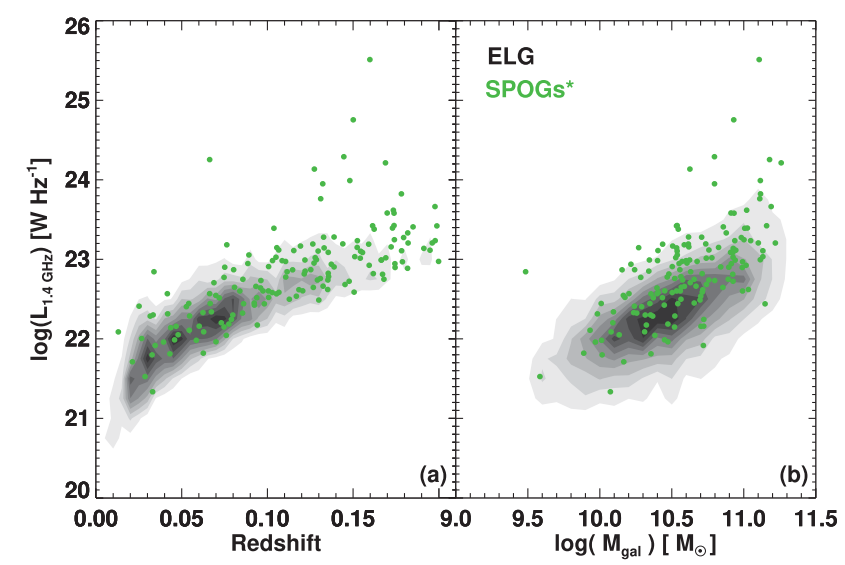

Figure 13. Radio luminosity FIRST-detected objects in the ELG sample (black) overlaid with SPOGs* (green points) as a function of redshift (left; a) and mass (right; b). Contours levels are in increments of $10 \%$ of the maximum.

Shabala 2015). The quintessential SPOG NGC 1266 is a case study for this phenomenon, with turbulent energy injection from the radio jets powering the shocks (Alatalo et al. 2011, 2014c). The fact that SPOGs* show slightly elevated radio detections compared to the rest of the ELG (and its subsamples; see Section 3.5) seems to indicate the possibility that radio jets could play a role in transitioning galaxies, but confirming this requires deeper and higher resolution radio data.

Mergers are thought to play a role in the morphological and color transition of poststarburst galaxies and these interactions could also fuel black hole growth in the form of quasar activity lasting up to $<10^{8}$ years (Canalizo \& Stockton 2000, 2013; Martini 2004; Mullaney et al. 2012). In order to be as massive as they are, all supermassive black holes are expected to go through powerful phases of accretion. Several studies have made connections between AGN/quasar phases and poststarburst activity (Brown et al. 2009; Wild et al. 2009; Cales et al. 2011, 2013; Kocevski et al. 2011; Yesuf et al. 2014; Cales \& Brotherton 2015), and though circumstantial, SPOGs could be the smoking gun revealing the AGN-host galaxy connection. Due to our shock boundaries, we are not sensitive to AGN activity producing the hardest radiation fields ([O III]/ $\mathrm{H} \beta>1.03$ ), though we would be able to identify objects with softer radiation fields (as is seen in some Type II quasars; Villar-Martín et al. 2008). ${ }^{19}$ The shock boundary might also allow us to observe systems that have recently undergone quasar activity, given both the stellar populations of quasars found by Canalizo \& Stockton (2013), as the AGN "flickering" time variability arguments of Schawinski et al. (2015) suggest that the short timescale AGN events could be caught using the SPOG criteria.

Galaxies falling into clusters or in group environments are also known to be transforming, having rapidly truncated their SF (Dressler 1980; Dressler \& Gunn 1983; Johnson et al. 2007; Ko et al. 2013; Lee et al. 2015). Many of these systems have also been detected to have shocks traced by warm $\mathrm{H}_{2}$ (Sivanandam et al. 2010; Cluver et al. 2013). Given the simultaneity of shocked gas and truncated SF, shocked group galaxies and cluster galaxies are likely to be identified using the SPOG criterion, and some examples are very likely present in our sample.

It is possible that a subset of SPOGs is not transitioning from blue to red at all, but instead is being refueled by the accretion of cold gas, temporarily replenishing SF (Kannappan et al. 2009; McIntosh et al. 2014) and producing shocks due to the interaction between the accreted material and the in-situ ISM of the recipient galaxy. This catalyst was argued by Dressler et al. (2013) as the origin of poststarburst galaxies in both clusters as well as the field, having transitioned across the green valley in the opposite sense (though this phase is expected to be short-lived; Young et al. 2014; Appleton et al. 2014).

Most likely, the SPOG criterion has identified examples of all of these sub-populations of transitioning galaxies: some hosting AGN-driven outflows, some on the tail-end of merging, some transitioning in a group or cluster setting, some replenishing from accretion, and likely some that do not fit into any of these categories. It will take detailed studies of their

\footnotetext{
19 A secondary check that can be run on this population is searching for substantial $[\mathrm{O} \mathrm{II}]$ emission, which should separate the quasars from shockdominated objects.
} 
environments, morphologies, interaction histories, kinematics, interstellar media, SF histories, and AGN properties to understand the nature of objects in a SPOG phase.

In order to gain a deeper understanding of SPOGs, we then must compare how SPOGs relate to the broader class of quenching galaxies that may or may not have shocks. SPOGs* as a sample are an excellent starting point, pinpointing the rare objects that warrant further study, and opening the door to a deeper understanding of the initial conditions that trigger the transformation of galaxies from blue spirals to red early-types (and possibly back again).

\section{SUMMARY}

As galaxies age, they move from the blue cloud (starforming spirals) to the red sequence (quiescent early-types) in color while also transforming morphologically. A few case studies in the nearby universe seem to indicate that a population of galaxies are transitioning "quietly," with few outward signs of the transformation, and warrant further investigation. Using the OSSY catalog from SDSS DR7 and requiring robust detections of all diagnostic emission lines (the ELG sample), we combine Balmer absorption selection criteria with emission line ratios consistent with shocked emission (but excluding pure SF) to create the SPOGS. However, these objects can only be considered candidates until observations confirming the presence of shocks can take place. We summarize our findings below.

1. Traditional poststarburst searches, which use the presence of Balmer absorption and the absence of nebular ([O II] and/or $\mathrm{H} \alpha$ ) emission, are able to identify galaxies that have recently transformed morphologically, but miss a large subset of transitioning galaxies in which other mechanisms (such as shocks and AGNs) excite the [O II] and $\mathrm{H} \alpha$.

2. We show that $0.2 \%$ (1067 galaxies) of the OSSY continuum sample fit the SPOGs criteria, which is comparable to catalogs of completely passive poststarburst galaxies, indicating that galaxies harboring powerful sources of ionization from AGN, LINERs and/or shocks are also an important contributor to the poststarburst class. SPOGs* are seen to be in the green valley, though with bluer colors than E+A-selected OSSY galaxies, suggestive of being in an earlier stage of transition.

3. The Na I D properties of SPOGs* are unique among the subsamples studied, showing a significant population of enhanced Na I D objects, suggesting that SPOGs* contain interstellar $\mathrm{NaID}$, which might imply the presence of galactic winds.

4. The SPOG $^{*}$ subsample has a $13 \%$ radio detection rate (the highest in the ELG, along with LINERs), suggesting that many SPOGs* host AGNs, although other origins for the radio emission must be ruled out first.

5. It is likely that the SPOG criteria have sampled a heterogeneous set of transitioning objects, including those with AGN-driven outflows, objects undergoing mergers, quasar hosts, galaxies entering clusters, in group environments, replenished ETG, as well as none of the above. Further studies of this SPOG* sample will be able to confirm whether the emission line ratios trace shocks, sample the morphologies and environments that SPOGs* inhabit, measure the AGN power and SF rate, and determine the ISM properties of objects undergoing a SPOG phase, to put a limit on the relative importance of each catalyst to the SPOG phenomenon.

K.A. and S.L.C. thank the thorough, thoughtful, and expert recommendations from the anonymous referee, which have vastly improved this manuscript. K.A. also thanks Alan Dressler for conversations about previously made assumptions, adding further depth to the manuscript. K.A. is supported through Hubble Fellowship grant \#HST-HF2-51352.001 awarded by the Space Telescope Science Institute, which is operated by the Association of Universities for Research in Astronomy, Inc., for NASA, under contract NAS5-26555. S.L.C. was supported by ALMA-CONICYT program 31110020. Partial support was provided to K.A. and P.N.A. by NASA observations through a contract issued by the Jet Propulsion Laboratory, California Institute of Technology under a contract with NASA. K.N. acknowledges support from NASA through the Spitzer Space Telescope. AMM and LJK acknowledge the support of the Australian Research Council (ARC) through Discovery project DP130103925.

Funding for SDSS-III has been provided by the Alfred P. Sloan Foundation, the Participating Institutions, the National Science Foundation, and the U.S. Department of Energy Office of Science. The SDSS-III web site is http://www.sdss3.org/. The National Radio Astronomy Observatory is a facility of the National Science Foundation operated under cooperative agreement by Associated Universities, Inc. This research has made use of the NASA/IPAC Extragalactic Database (NED) which is operated by the Jet Propulsion Laboratory, California Institute of Technology, under contract with the National Aeronautics and Space Administration.

\section{APPENDIX \\ INVESTIGATING THE POTENTIAL BIASES FOR THE ELG AND SPOGS}

With the ability to study millions of galaxies over cosmic time, astronomers using SDSS data have transformed the study of galaxy evolution. However, despite this success, fiber spectroscopy suffers from a major drawback: for nearby galaxies, a fiber is only able to target its center. Within the OSSY sample, the size of the $3^{\prime \prime}$ SDSS fiber varies in physical scale upon the galaxy by over two orders of magnitude, based on the redshift that is being investigated. For this reason, we must discuss possible aperture effects that are present in both the ELG and the SPOG* ${ }^{*}$ sample.

\section{A.1. The Effect of Requiring Bright [O I] Emission to the Sample}

First, we explored whether the emission line selection criterion (and thus the relative fractions of diagnostic classifications) was biased by redshift, due to requiring the detection of a weak line ([O I $]$ ). There were 280,378 objects that met the cuts in all other lines $\left(\mathrm{H} \alpha, \mathrm{H} \beta,\left[\mathrm{N}_{\mathrm{II}}\right],\left[\mathrm{S}_{\mathrm{II}}\right]\right.$, and $[\mathrm{O}$ III]).

We plotted the distributions of redshifts in Figure 14(a), which shows that the [O I] cut slightly favors lower redshift objects, consistent with a slight Malmquist bias (see: Appendix A.2). The average redshift of the objects where no [O I] cut was applied is $z=0.090$, compared to [O I] cuts, with $z=0.080$. 

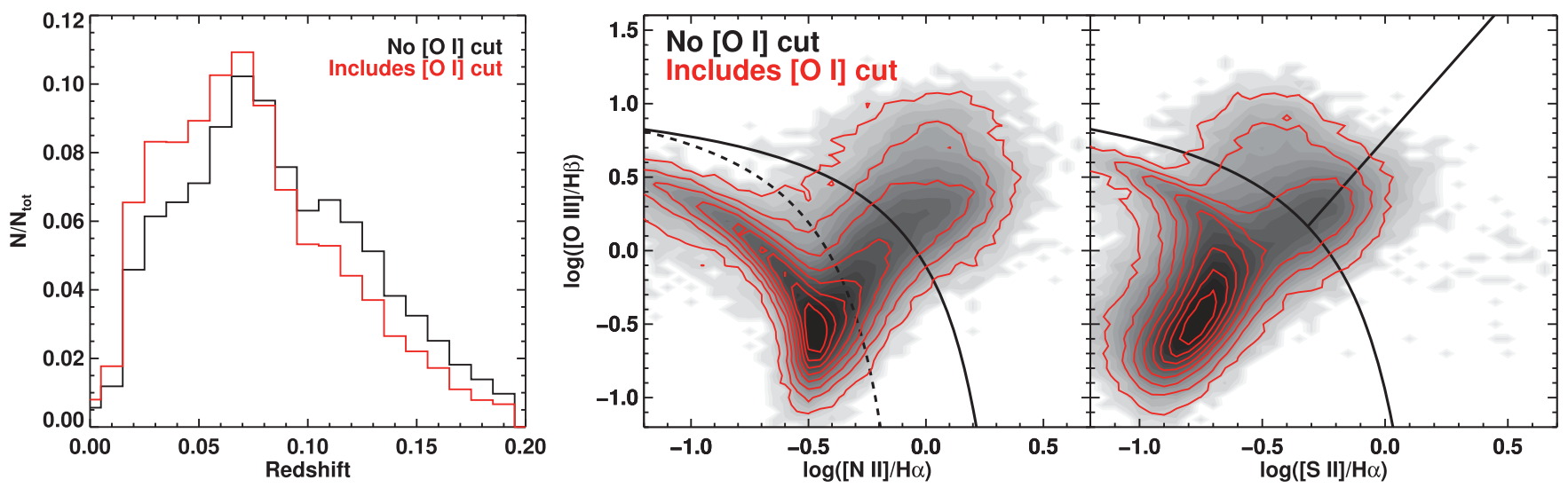

Figure 14. (Left): The redshift distributions of OSSY galaxies that pass the continuum $\mathrm{S} / \mathrm{N}$ cuts, comparing the galaxies detected in $\mathrm{H} \alpha, \mathrm{H} \beta$, $[\mathrm{O} \mathrm{III}],[\mathrm{S} \Pi]$, and $[\mathrm{N} \Pi]$ (black) to those that pass the $\left[\mathrm{O}_{\mathrm{I}}\right]$ cut (red). (Right): Comparison of the $[\mathrm{N} \mathrm{II}] / \mathrm{H} \alpha$ and $[\mathrm{S} \mathrm{II}] / \mathrm{H} \alpha$ for objects with (red) and without (grayscale) $[\mathrm{O}$ I] cuts.
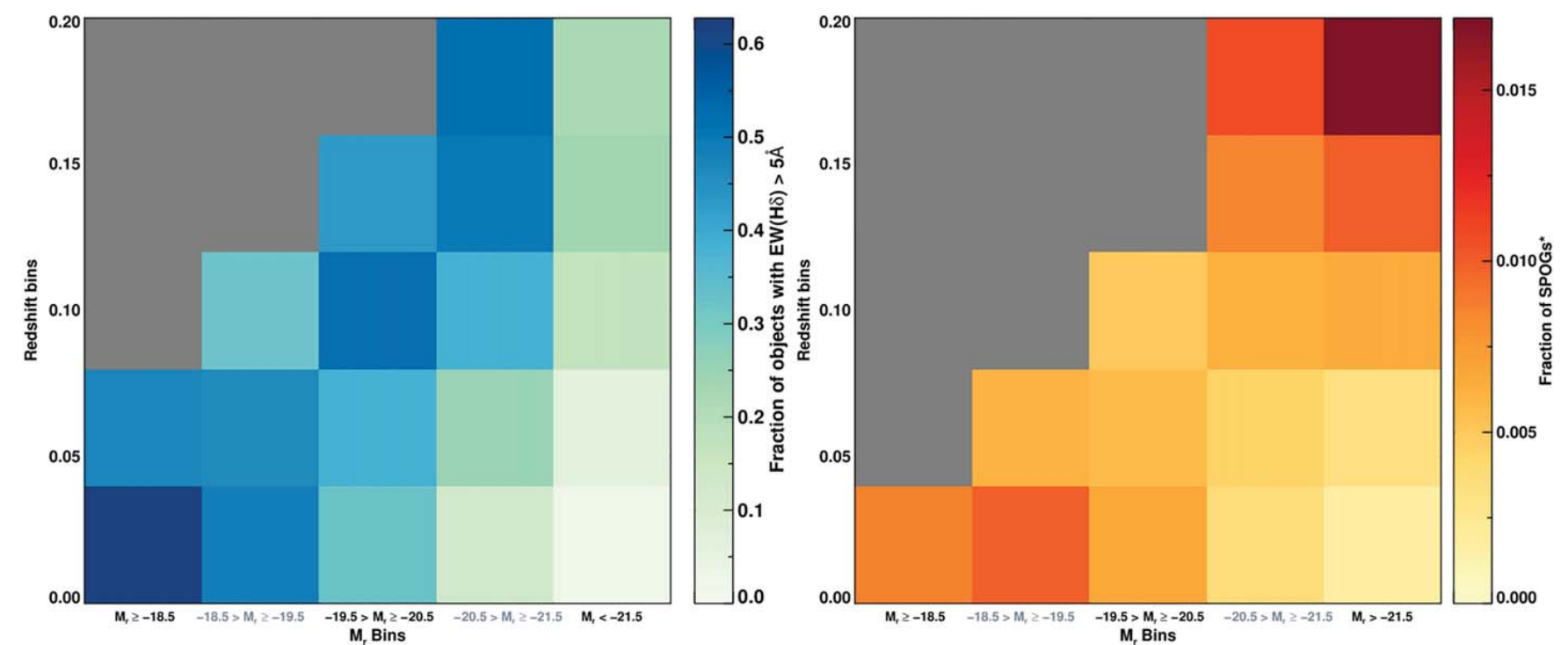

Figure 15. (Left): Detections of galaxies with $\mathrm{EW}(\mathrm{H} \delta)>5 \AA$ as a function of absolute $r$-band magnitude and redshift. The fraction shows both a trend in $M_{r}$, and a trend in redshift, with the smallest $M_{r}$ lowest redshift bin containing the most objects with $\mathrm{EW}(\mathrm{H} \delta)>5 \AA$, and the lowest detection rate in the largest $M_{r}$ bins. Regions shaded gray contain no objects. (Right): The SPOG* fraction as a function of absolute $r$-band magnitude and redshift. Interestingly, the SPOG* detection rate does not appear to show trends with redshift below $M_{r}<-20.5$, though the overall detection rate does not vary by more than a factor of 3 per bin. The largest $M_{r}$, highest redshift bins have the highest SPOG* identification rate, with a secondary peak at low redshift, small $M_{r}$ objects.

Figure 14(b) shows the $[\mathrm{N} \mathrm{II}] / \mathrm{H} \alpha$ and $[\mathrm{S}$ II] $/ \mathrm{H} \alpha$ versus $[\mathrm{O} \mathrm{III}] / \mathrm{H} \beta$ line diagnostics without the $\left[\mathrm{O}_{\mathrm{I}}\right]$ cut in grayscale, compared to applying the [O I] cut (red). The [O I] cut does not prefer specific segments of the line diagnostic diagrams. We therefore do not believe that our selection for the [O I] line imposes biases significant enough to impact our conclusions.

\section{A.2. Aperture and Malmquist Bias}

The small central aperture did find objects classified as SPOGs* that had extended SF (see Section 2.5). To investigate the magnitude of this issue, we plotted the $\operatorname{EW}(\mathrm{H} \delta)>5 \AA$ and SPOG $^{*}$ detection rate as a function of $M_{r}$ and redshift, shown in Figure 15 (note: Figure 5 shows that small $M_{r}$, high redshift objects are not found in the ELG). The identification fraction of $\mathrm{EW}(\mathrm{H} \delta)>5 \AA$ objects strongly depends both on $M_{r}$ and redshift, with large $M_{r}$ objects being less likely to have strong Balmer absorption. At the smallest $M_{r}$, the $\mathrm{EW}(\mathrm{H} \delta)>$ $5 \AA$ Araction decreases slightly with redshift, possibly due to a number of low mass dwarfs that fit the ELG criterion, but this trend reverses as $M_{r}$ grows larger, with increasing detection rates among the higher redshift ELG objects. It is very likely that this is due to the SDSS fiber picking up larger areas of the star-forming disk (as opposed to the bulge).

The SPOG ${ }^{*}$ detection versus $M_{r}$ and redshift (Figure 15(b)) has two peaks, one toward the larger $M_{r}$, highest redshift objects, and one near the smallest $M_{r}$, lowest redshift, though the SPOG* ${ }^{*}$ identification rate does not change by more than a factor of 3 through the sample. While aperture effects could explain this peak, other effects (including Malmquist bias and metallicity effects) might also explain the slightly elevated detection rate in the small $M_{r}$, low redshift bin.

Malmquist bias (the proclivity to sample the brightest objects; Malmquist 1925) is well known in sample selections, especially those with flux cutoffs. The most obvious manifestation is seen in Figure 5, showing that the average absolute magnitude within the ELG increases with redshift. It is possible that the high detection rate of SPOGs* in the highest mass, 


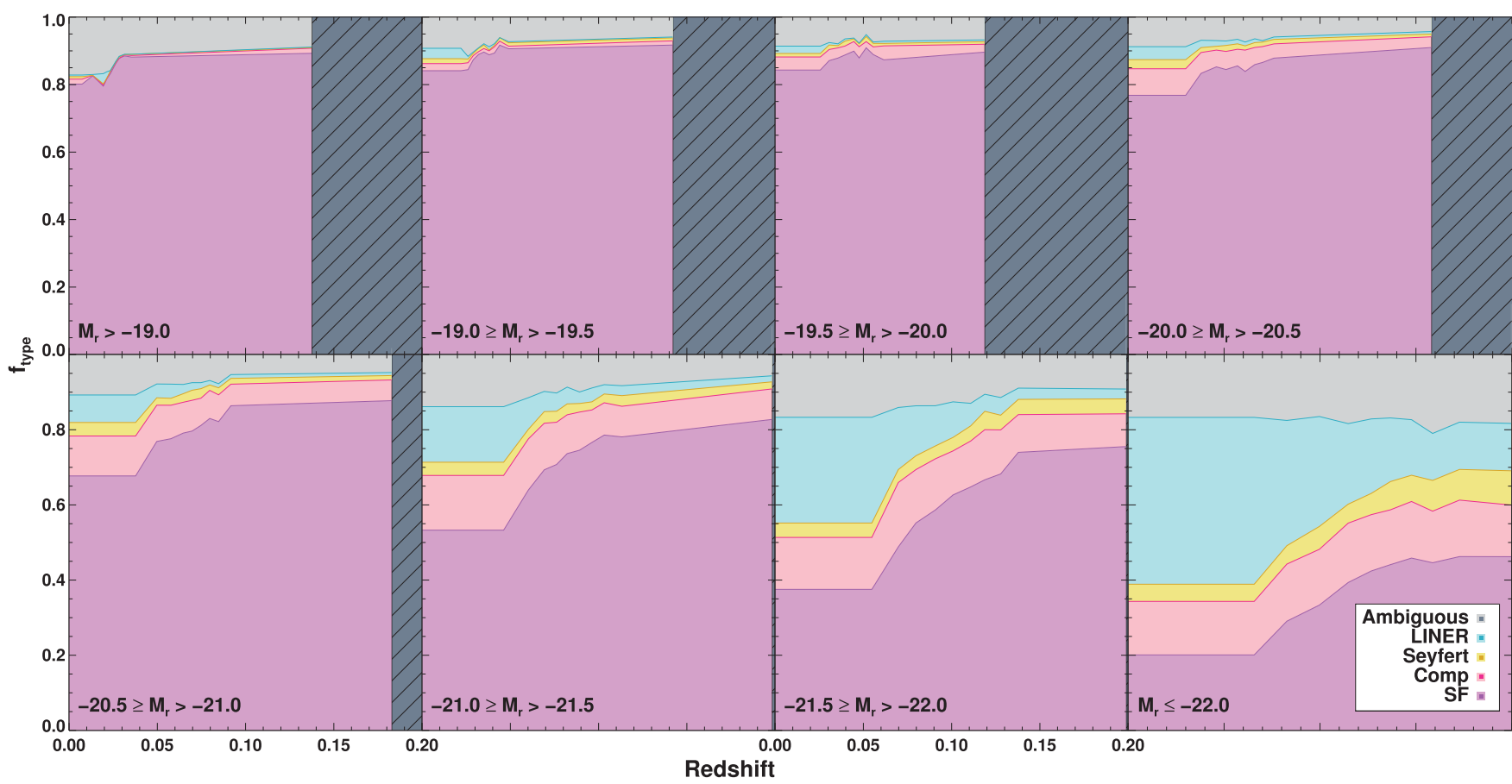

Figure 16. The line diagnostic classifications from Kewley et al. (2006) as a function of redshift in eight distinct mass bins, from lowest mass to highest. The redshift bins were determined by sub-dividing all galaxies in each mass category evenly between 10 separate redshift bins. The redshift bin point is placed at the high reshift point of each bin. This means that all redshift bins have the same number of total objects. The dark gray blocked region of the plot represents redshift space where no ELG objects within the mass bin are detected. In the cases of the all but the highest mass bin, the majority of objects are star-forming, with a significant minority falling into the ambiguous category.

highest redshift objects is in part due to Malmquist bias, with the most massive objects at the highest redshift also tending to sample a rarer, brighter subsample. In particular, the fact that there is a significant drop in the fraction of $\mathrm{EW}(\mathrm{H} \delta)>5 \AA$ objects at the high mass, high redshift end seems to indicate that the ELG is sampling other bright sources of emission, given that the high mass end of the galaxy distribution function has significantly fewer star-forming galaxies (Schawinski et al. 2014; Ogle et al. 2016). Given that Malmquist bias has manifested in the detection fractions of Balmer absorbing systems (and possible SPOGs), it may also impact the relative proportion of line diagnostic types that are detected within the ELG.

To investigate this, we plotted the redshift-dependent line diagnostic classification in Figure 16. In most cases, the mass has a significant effect on the fractional representation of each line diagnostic classification. The 4 lowest mass bins contain at least $75 \%$ star-forming classified objects, with a substantial fraction of objects also classified as ambiguous at the lowest redshifts. At these low redshifts, the ELG would be able to detect dwarf star-forming galaxies. These galaxies have low metallicities, which can pump the [O III] compared to $\mathrm{H} \beta$ emission, and suppress [N $\left.\mathrm{N}_{\mathrm{II}}\right]$ compared to $\mathrm{H} \alpha$ emission, pushing the emission line diagnostic into the Seyfert class (Kewley et al. 2013a, 2013b). This would then create a disagreement between the various line diagnostics, thus making the resultant integrated classification ambiguous.

AGN line classification appears to be consistent to $z<0.3$ (LaMassa et al. 2012). The AGN fraction within the ELG does not change much with redshift over the different mass bins, although there are more AGNs identified as a function of absolute magnitude, consistent with observations that AGNs lie in massive host galaxies (Kauffmann et al. 2003). The detection rate of composite objects also does not appear to change with redshift (and thus galactic area subtended by the SDSS fiber), consistent with the results of LaMassa et al. (2013) suggesting that the circumnuclear SF in AGN hosts tends to be compact $(<1.7 \mathrm{kpc})$.

In the two highest mass bins, there are some trends with redshift, including a larger percentage of objects with LINER classifications at low redshift. This is in agreement with the behavior of LINERs from Kewley et al. (2006), as LINER-like emission tends to be weak (Yan et al. 2006), and located in massive galaxies (Sarzi et al. 2006, 2010), thus the more nearby massive LINER host galaxies are the most likely to surpass the emission line thresholds of the ELG sample. Since LINER emission is thought to be widespread in massive galaxies (Sarzi et al. 2006, 2010; Capetti \& Baldi 2011), one might expect the detection rate to increase with redshift (as is seen in starforming objects) due to the change in the physical scale of the SDSS fiber, but the intrinsic weakness of the emission seems to be a stronger effect in our LINER detection rate.

Overall, this likely means that the SPOG* catalog has notable biases, namely, a slight aperture bias and Malmquist bias. The aperture bias allows some low redshift objects to be classified as SPOGs* despite hosting extended SF, though it might also work in the other direction (as is seen with $\mathrm{EW}(\mathrm{H} \delta)>5 \AA$ objects in Figure 15) when the SDSS fiber is able to sample larger portions of the star-forming disk (rather than purely the bulge light). The SPOG* sample also suffers from Malmquist bias, with the detection rate increasing as a function of both absolute magnitude and redshift, so SPOGs* have been more commonly found among the brightest and rarest of the objects in our sample. Despite these biases, the 
SPOG criterion seems to have uncovered a new sample of transitioning galaxies, though future surveys and applications of the SPOG catalog should keep these biases in mind.

\section{REFERENCES}

Aalto, S., Costagliola, F., Muller, S., et al. 2015, A\&A, submitted (arXiv: 1510.08827)

Abazajian, K. N., Adelman-McCarthy, J. K., the SDSS Collaboration, et al. 2009, ApJS, 182, 543

Ahn, C. P., Alexandroff, R., the SDSS Collaboration, et al. 2012, ApJS, 203, 21

Alam, S., Albareti, F. D., Allende Prieto, C., et al. 2015, ApJS, 219, 12

Alatalo, K. 2015, ApJL, 801, L17

Alatalo, K., Appleton, P. N., Lisenfeld, U., et al. 2014a, ApJ, 795, 159

Alatalo, K., Appleton, P. N., Lisenfeld, U., et al. 2015a, ApJ, 812, 117

Alatalo, K., Blitz, L., Young, L. M., et al. 2011, ApJ, 735, 88

Alatalo, K., Cales, S. L., Appleton, P. N., et al. 2014b, ApJL, 794, L13

Alatalo, K., Lacy, M., Lanz, L., et al. 2015b, ApJ, 798, 31

Alatalo, K., Nyland, K., Graves, G., et al. 2014c, ApJ, 780, 186

Allen, M. G., Groves, B. A., Dopita, M. A., et al. 2008, ApJS, 178, 20

Appleton, P. N., Fadda, D. T., Marleau, F. R., et al. 2004, ApJS, 154, 147

Appleton, P. N., Guillard, P., Boulanger, F., et al. 2013, ApJ, 777, 66

Appleton, P. N., Mundell, C., Bitsakis, T., et al. 2014, ApJ, 797, 117

Appleton, P. N., Xu, K. C., Reach, W., et al. 2006, ApJL, 639, L51

Armus, L., Heckman, T. M., \& Miley, G. K. 1990, ApJ, 364, 471

Baade, W. 1958, RA, 5, 3

Baldry, I. K., Glazebrook, K., Brinkmann, J., et al. 2004, ApJ, 600, 681

Baldwin, J. A., Phillips, M. M., \& Terlevich, R. 1981, PASP, 93, 5

Becker, R. H., White, R. L., \& Helfand, D. J. 1995, ApJ, 450, 559

Bekki, K. 1998, ApJL, 502, L133

Bekki, K., Couch, W. J., \& Shioya, Y. 2002, ApJ, 577, 651

Bell, E. F., McIntosh, D. H., Katz, N., \& Weinberg, M. D. 2003, ApJS, 149, 289

Bell, E. F., van der Wel, A., Papovich, C., et al. 2012, ApJ, 753, 167

Bell, E. F., Zheng, X. Z., Papovich, C., et al. 2007, ApJ, 663, 834

Best, P. N., \& Heckman, T. M. 2012a, MNRAS, 421, 1569

Best, P. N., \& Heckman, T. M. 2012b, yCat, 742, 11569

Best, P. N., Kauffmann, G., Heckman, T. M., et al. 2005, MNRAS, 362, 25

Bîrzan, L., McNamara, B. R., Nulsen, P. E. J., et al. 2008, ApJ, 686, 859

Black, J. H., \& van Dishoeck, E. F. 1987, ApJ, 322, 412

Blanton, M. R., \& Moustakas, J. 2009, ARA\&A, 47, 159

Braglia, F. G., Pierini, D., Biviano, A., \& Böhringer, H. 2009, A\&A, 500, 947

Brown, M. J. I., Moustakas, J., Caldwell, N., et al. 2009, ApJ, 703, 150

Bruzual, G., \& Charlot, S. 2003, MNRAS, 344, 1000

Bundy, K., Bershady, M. A., Law, D. R., et al. 2015, ApJ, 798, 7

Cales, S. L., \& Brotherton, M. S. 2015, MNRAS, 449, 2374

Cales, S. L., Brotherton, M. S., Shang, Z., et al. 2011, ApJ, 741, 106

Cales, S. L., Brotherton, M. S., Shang, Z., et al. 2013, ApJ, 762, 90

Canalizo, G., \& Stockton, A. 2000, AJ, 120, 1750

Canalizo, G., \& Stockton, A. 2013, ApJ, 772, 132

Cano-Díaz, M., Maiolino, R., Marconi, A., et al. 2012, A\&A, 537, L8

Capetti, A., \& Baldi, R. D. 2011, A\&A, 529, A126

Cappellari, M., \& Emsellem, E. 2004, PASP, 116, 138

Cattaneo, A., Dekel, A., Devriendt, J., Guiderdoni, B., \& Blaizot, J. 2006, MNRAS, 370, 1651

Cen, R., Pop, A. R., \& Bahcall, N. A. 2014, PNAS, 111, 7914

Chen, Y.-M., Tremonti, C. A., Heckman, T. M., et al. 2010, AJ, 140, 445

Chilingarian, I. V., \& Zolotukhin, I. Y. 2012, MNRAS, 419, 1727

Chung, A., van Gorkom, J. H., Kenney, J. D. P., Crowl, H., \& Vollmer, B. 2009, AJ, 138, 1741

Cicone, C., Maiolino, R., Gallerani, S., et al. 2015, A\&A, 574, A14

Cicone, C., Maiolino, R., Sturm, E., et al. 2014, A\&A, 562, A21

Ciotti, L., \& Ostriker, J. P. 2007, ApJ, 665, 1038

Ciotti, L., Ostriker, J. P., \& Proga, D. 2010, ApJ, 717, 708

Cluver, M. E., Appleton, P. N., Boulanger, F., et al. 2010, ApJ, 710, 248

Cluver, M. E., Appleton, P. N., Ogle, P., et al. 2013, ApJ, 765, 93

Condon, J. J. 1992, ARA\&A, 30, 575

Condon, J. J., Cotton, W. D., Greisen, E. W., et al. 1998, AJ, 115, 1693

Costa, T., Sijacki, D., \& Haehnelt, M. G. 2014, MNRAS, 444, 2355

Croom, S. M., Lawrence, J. S., Bland-Hawthorn, J., et al. 2012, MNRAS, 421,872

Darg, D. W., Kaviraj, S., Lintott, C. J., et al. 2010, MNRAS, 401, 1043

Davies, R. L., Rich, J. A., Kewley, L. J., \& Dopita, M. A. 2014, MNRAS, 439,3835
Davis, T. A., Alatalo, K., Sarzi, M., et al. 2011, MNRAS, 417, 882

Davis, T. A., Krajnović, D., McDermid, R. M., et al. 2012, MNRAS, 426, 1574

Davis, T. A., Young, L. M., Crocker, A. F., et al. 2014, MNRAS, 444, 3427

Di Matteo, T., Springel, V., \& Hernquist, L. 2005, Natur, 433, 604

Dopita, M. A., Groves, B. A., Fischera, J., et al. 2005, ApJ, 619, 755

Dopita, M. A., \& Sutherland, R. S. 1995, ApJ, 455, 468

Dopita, M. A., \& Sutherland, R. S. 1996, ApJS, 102, 161

Dressler, A. 1980, ApJ, 236, 351

Dressler, A., \& Gunn, J. E. 1983, ApJ, 270, 7

Dressler, A., Oemler, A., Jr., Poggianti, B. M., et al. 2013, ApJ, 770, 62

Faber, S. M., Willmer, C. N. A., Wolf, C., et al. 2007, ApJ, 665, 265

Falkenberg, M. A., Kotulla, R., \& Fritze, U. 2009, MNRAS, 397, 1940

Farage, C. L., McGregor, P. J., Dopita, M. A., et al. 2010, ApJ, 724, 267

Feruglio, C., Maiolino, R., Piconcelli, E., et al. 2010, A\&A, 518, L155

Fogarty, L. M. R., Bland-Hawthorn, J., Croom, S. M., et al. 2012, ApJ, 761, 169

Forster, K., Rich, R. M., \& McCarthy, J. K. 1995, ApJ, 450, 74

Gabor, J. M., \& Bournaud, F. 2014, MNRAS, 441, 1615

García-Burillo, S., Combes, F., Usero, A., et al. 2014, A\&A, 567, A125

Goto, T. 2005, MNRAS, 357, 937

Goto, T. 2007, MNRAS, 377, 1222

Guillard, P., Boulanger, F., Lehnert, M. D., et al. 2015, A\&A, 574, A32

Guillard, P., Boulanger, F., Pineau Des Forêts, G., \& Appleton, P. N. 2009, A\&A, 502, 515

Guillard, P., Boulanger, F., Pineau Des Forêts, G., et al. 2012, ApJ, 749, 158

Gunn, J. E., \& Gott, J. R., III 1972, ApJ, 176, 1

Harrison, C. M., Alexander, D. M., Mullaney, J. R., \& Swinbank, A. M. 2014, MNRAS, 441, 3306

Heckman, T. M., Armus, L., \& Miley, G. K. 1990, ApJS, 74, 833

Heckman, T. M., Lehnert, M. D., Strickland, D. K., \& Armus, L. 2000, ApJS, 129,493

Hickson, P. 1997, ARA\&A, 35, 357

Ho, I.-T., Kewley, L. J., Dopita, M. A., et al. 2014, MNRAS, 444, 3894

Ho, I. T., Medling, A. M., Bland-Hawthorn, J., et al. 2016, MNRAS, 457, 1257

Hogg, D. W., Masjedi, M., Berlind, A. A., et al. 2006, ApJ, 650, 763

Holmberg, E. 1958, MeLuS, 136, 1

Hopkins, P. F., Hernquist, L., Cox, T. J., \& Kereš, D. 2008, ApJS, 175, 356

Hopkins, P. F., Hernquist, L., Cox, T. J., et al. 2006, ApJS, 163, 1

Hubble, E. P. 1926, ApJ, 64, 321

Inami, H., Armus, L., Charmandaris, V., et al. 2013, ApJ, 777, 156

Ivezić, Ž, Menou, K., Knapp, G. R., et al. 2002, AJ, 124, 2364

Jeong, H., Yi, S. K., Kyeong, J., et al. 2013, ApJS, 208, 7

Johnson, K. E., Hibbard, J. E., Gallagher, S. C., et al. 2007, AJ, 134, 1522

Kannappan, S. J., Guie, J. M., \& Baker, A. J. 2009, AJ, 138, 579

Kauffmann, G., Heckman, T. M., Tremonti, C., et al. 2003, MNRAS, 346, 1055

Kehrig, C., Monreal-Ibero, A., Papaderos, P., et al. 2012, A\&A, 540, A11

Kewley, L. J., Dopita, M. A., Leitherer, C., et al. 2013a, ApJ, 774, 100

Kewley, L. J., Geller, M. J., \& Jansen, R. A. 2004, AJ, 127, 2002

Kewley, L. J., Groves, B., Kauffmann, G., \& Heckman, T. 2006, MNRAS, 372,961

Kewley, L. J., Maier, C., Yabe, K., et al. 2013b, ApJL, 774, L10

Ko, J., Hwang, H. S., Lee, J. C., \& Sohn, Y.-J. 2013, ApJ, 767, 90

Kocevski, D. D., Lemaux, B. C., Lubin, L. M., et al. 2011, ApJL, 737, L38

Krug, H. B., Rupke, D. S. N., \& Veilleux, S. 2010, ApJ, 708, 1145

LaMassa, S. M., Heckman, T. M., Ptak, A., \& Urry, C. M. 2013, ApJ, 765, L33

LaMassa, S. M., Heckman, T. M., Ptak, A., et al. 2012, ApJ, 758, 1

Lanz, L., Hayward, C. C., Zezas, A., et al. 2014, ApJ, 785, 39

Lanz, L., Ogle, P. M., Alatalo, K., \& Appleton, P. N. 2015, ApJ, in press (arXiv:1511.05968)

Lee, G.-H., Hwang, H. S., Lee, M. G., et al. 2015, ApJ, 800, 80

Lesaffre, P., Pineau des Forêts, G., \& Godard, B. 2013, A\&A, 550, A106

Lilly, S. J., Carollo, C. M., Pipino, A., Renzini, A., \& Peng, Y. 2013, ApJ, 772,119

Lisenfeld, U., \& Völk, H. J. 2010, A\&A, 524, A27

Lotz, J. M., Jonsson, P., Cox, T. J., et al. 2011, ApJ, 742, 103

Malmquist, K. G. 1925, MeLuF, 106, 1

Martig, M., Bournaud, F., Teyssier, R., \& Dekel, A. 2009, ApJ, 707, 250

Martig, M., Crocker, A. F., Bournaud, F., et al. 2013, MNRAS, 432, 1914

Martin, C. L. 2006, ApJ, 647, 222

Martini, P. 2004, in Coevolution of Black Holes and Galaxies, ed. L. C. Ho (Cambridge: Cambridge Univ. Press), 169

McConnell, N. J., Lu, J. R., \& Mann, A. W. 2015, ApJ, 821, 39

McIntosh, D. H., Wagner, C., Cooper, A., et al. 2014, MNRAS, 442, 533

Mihos, J. C. 1995, ApJL, 438, L75 
Monreal-Ibero, A., Arribas, S., \& Colina, L. 2006, ApJ, 637, 138

Monreal-Ibero, A., Arribas, S., Colina, L., et al. 2010, A\&A, 517, A28

Moore, B., Katz, N., Lake, G., Dressler, A., \& Oemler, A. 1996, Natur, 379,613

Mouri, H., Kawara, K., \& Taniguchi, Y. 2000, ApJ, 528, 186

Mullaney, J. R., Daddi, E., Béthermin, M., et al. 2012, ApJL, 753, L30

Murray, N., Martin, C. L., Quataert, E., \& Thompson, T. A. 2007, ApJ, 660,211

Nesvadba, N. P. H., Lehnert, M. D., De Breuck, C., Gilbert, A. M., \& van Breugel, W. 2008, A\&A, 491, 407

Noeske, K. G., Weiner, B. J., Faber, S. M., et al. 2007, ApJL, 660, L43

Nyland, K., Alatalo, K., Wrobel, J. M., et al. 2013, ApJ, 779, 173

Ogle, P. M., Lanz, L., Nader, C., \& Helou, G. 2016, ApJ, 817, 109

Oh, K., Sarzi, M., Schawinski, K., \& Yi, S. K. 2011, ApJS, 195, 13

Owen, F. N., \& Laing, R. A. 1989, MNRAS, 238, 357

Pattarakijwanich, P., Strauss, M. A., Ho, S., \& Ross, N. P. 2014, ApJ, submitted (arXiv:1410.7394)

Peng, Y.-j., Lilly, S. J., Kovač, K., et al. 2010, ApJ, 721, 193

Peterson, B. W., Appleton, P. N., Helou, G., et al. 2012, ApJ, 751, 11

Quintero, A. D., Hogg, D. W., Blanton, M. R., et al. 2004, ApJ, 602, 190

Rasmussen, J., Ponman, T. J., Verdes-Montenegro, L., Yun, M. S., \& Borthakur, S. 2008, MNRAS, 388, 1245

Rich, J. A., Dopita, M. A., Kewley, L. J., \& Rupke, D. S. N. 2010, ApJ, 721,505

Rich, J. A., Kewley, L. J., \& Dopita, M. A. 2011, ApJ, 734, 87

Rich, J. A., Kewley, L. J., \& Dopita, M. A. 2014, ApJL, 781, L12

Rich, J. A., Kewley, L. J., \& Dopita, M. A. 2015, ApJS, 221, 28

Rupke, D. S., Veilleux, S., \& Sanders, D. B. 2005, ApJS, 160, 115

Rupke, D. S. N., \& Veilleux, S. 2011, ApJL, 729, L27

Rupke, D. S. N., \& Veilleux, S. 2015, ApJ, 801, 126

Sánchez, S. F., Kennicutt, R. C., Gil de Paz, A., et al. 2012, A\&A, 538, A8

Sarzi, M., Falcón-Barroso, J., Davies, R. L., et al. 2006, MNRAS, 366, 1151

Sarzi, M., Kaviraj, S., Nedelchev, B., et al. 2016, MNRAS, 456, L25

Sarzi, M., Shields, J. C., Schawinski, K., et al. 2010, MNRAS, 402, 2187

Schawinski, K., Koss, M., Berney, S., \& Sartori, L. F. 2015, MNRAS, 451,2517

Schawinski, K., Urry, C. M., Simmons, B. D., et al. 2014, MNRAS, 440, 889

Schoenmakers, A. P., de Bruyn, A. G., Röttgering, H. J. A., van der Laan, H., \& Kaiser, C. R. 2000, MNRAS, 315, 371

Shull, J. M., \& Beckwith, S. 1982, ARA\&A, 20, 163

Sivanandam, S., Rieke, M. J., \& Rieke, G. H. 2010, ApJ, 717, 147
Snyder, G. F., Cox, T. J., Hayward, C. C., Hernquist, L., \& Jonsson, P. 2011, ApJ, 741, 77

Soto, K. T., Martin, C. L., Prescott, M. K. M., \& Armus, L. 2012, ApJ, 757, 86 Spergel, D. N., Bean, R., Doré, O., et al. 2007, ApJS, 170, 377 Springel, V., Di Matteo, T., \& Hernquist, L. 2005, ApJL, 620, L79

Stickley, N. R., \& Canalizo, G. 2014, ApJ, 786, 12

Strateva, I., Ivezić, Ž., Knapp, G. R., et al. 2001, AJ, 122, 1861

Sturm, E., González-Alfonso, E., Veilleux, S., et al. 2011, ApJL, 733, L16

Taylor, M. B. 2005, in ASP Conf. Ser. 347, Astronomical Data Analysis Software and Systems XIV, ed. P. Shopbell, M. Britton, \& R. Ebert (San Francisco, CA: ASP), 29

Tinsley, B. M. 1978, ApJ, 222, 14

Toomre, A., \& Toomre, J. 1972, ApJ, 178, 623

Treister, E., Schawinski, K., Urry, C. M., \& Simmons, B. D. 2012, ApJL, 758, L39

Turner, R. J., \& Shabala, S. S. 2015, ApJ, 806, 59

Van Wassenhove, S., Volonteri, M., Mayer, L., et al. 2012, ApJL, 748, L7

Vazdekis, A., Sánchez-Blázquez, P., Falcón-Barroso, J., et al. 2010, MNRAS, 404, 1639

Veilleux, S., \& Osterbrock, D. E. 1987, ApJS, 63, 295

Verdes-Montenegro, L., Yun, M. S., Williams, B. A., et al. 2001, A\&A, 377, 812

Villar Martín, M., Emonts, B., Humphrey, A., Cabrera Lavers, A., \& Binette, L. 2014, MNRAS, 440, 3202

Villar-Martín, M., Humphrey, A., Martínez-Sansigre, A., et al. 2008, MNRAS, 390, 218

Vogt, F. P. A., Dopita, M. A., Borthakur, S., et al. 2015, MNRAS, 450, 2593

Vogt, F. P. A., Dopita, M. A., \& Kewley, L. J. 2013, ApJ, 768, 151

Walker, L. M., Johnson, K. E., Gallagher, S. C., et al. 2010, AJ, 140, 1254

Wild, V., Walcher, C. J., Johansson, P. H., et al. 2009, MNRAS, 395, 144

Wong, O. I., Schawinski, K., Kaviraj, S., et al. 2012, MNRAS, 420, 1684

Wright, E. L., Eisenhardt, P. R. M., Mainzer, A. K., et al. 2010, AJ, 140, 1868

Wuyts, S., Förster Schreiber, N. M., van der Wel, A., et al. 2011, ApJ, 742, 96 Yan, R., \& Blanton, M. R. 2012, ApJ, 747, 61

Yan, R., Newman, J. A., Faber, S. M., et al. 2006, ApJ, 648, 281

Yang, Y., Zabludoff, A. I., Zaritsky, D., Lauer, T. R., \& Mihos, J. C. 2004, ApJ, 607, 258

Yang, Y., Zabludoff, A. I., Zaritsky, D., \& Mihos, J. C. 2008, ApJ, 688, 945

Yesuf, H. M., Faber, S. M., Trump, J. R., et al. 2014, ApJ, 792, 84

Young, L. M., Scott, N., Serra, P., et al. 2014, MNRAS, 444, 3408

Zabludoff, A. I., Zaritsky, D., Lin, H., et al. 1996, ApJ, 466, 104

Zakamska, N. L., Hamann, F., \& Pâris, I. 2016, MNRAS, 459, 3144 